\title{
Wirbellose Meerestiere als Parasiten, Kommensalen oder Symbionten in oder an Scyphomedusen
}

\author{
M. E. THIEL \\ Zoologisches Institut und Zoologisches Museum der Universität Hamburg; \\ Hamburg 13, Bundesrepublik Deutschland
}

\begin{abstract}
Marine invertebrates as parasites, commensales or symbionts associated with Scyphomedusae. Observations on invertebrates living in or on Scyphomedusae are compiled from literature. 51 species of 28 genera are listed as hosts for different taxa (Actiniariae, Turbellaria, Trematoda, Cestoda, Nematoda, Crustacea, Echinodermata and Cephalopoda). The relation of these invertebrates to their hosts is discussed and characterized as parasitism, commensalism or symbiosis. Trematodes and cestodes may be regarded as true parasites, with medusae as intermediate hosts and fishes as final hosts. The feeding on detritus from the mouthfrills may be interpreted as a cleaning symbiosis. The accociations of the recently discovered deepsea medusa Deepstaria enigmatica with the giant isopod Anuropus sp. and the rhizostome Catostylus ouwensi with the parasitic Ouwensia catostyli (the taxonomic position of which is still unclear) are described and discussed in detail.
\end{abstract}

\section{EINLEITUNG}

Uber das Vorkommen wirbelloser Tiere als Parasiten, Kommensalen oder Symbionten in oder an Scyphomedusen, deren Körper zum großen Teil aus mehr oder minder fester Gallerte besteht und nur sehr wenig fleischartige Substanz (Muskelgewebe, Geschlechtsorgane, ento- und ectodermale Epithelien) enthält, finden sich einige Mitteilungen bereits in der älteren Literatur über Scyphomedusen. So weist bereits BASTER (1762) darauf hin, daß in Cyanea capillata Parasiten oder Kommensalen vorkommen. Während diese Beobachtung erst etwa 100 Jahre später von VAN BENEDEN (1876) übernommen und dadurch bekannt wurde, teilte schon PERON (1801) den Fund einer Bandwurmlarve in einer Pbyllorbiza rosea, wohl der späteren Mastigias rosea, mit (Dollfuss, 1923, 1929). FABER (1829) stellte das Vorhandensein von kleinen parasitischen Crustaceen in den "Respirationshöhlen" (= Subgenitalhöhlen) von Aurelia aurita fest, und Котzeвue (1830) sah auf der berühmten Reise um die Welt auf der "Rubrik" auf dem Schirm einer "Pelagia mit der gelben Magenhaut" einen Cirripedier der Gattung Cineras sitzen. Im gleichen Jahre erwähnt Della Valle (1830) in der Beschreibung von Cassiopea borbonica ( = Cotylorbiza tuberculata) das Vorkommen von Distomeen, die er Distoma carinariae nennt. So ist bereits bis zum Ende des 1. Drittels des 19. Jahrhunderts das Vorkommen von Trematoden, Cestoden, Crusta- 


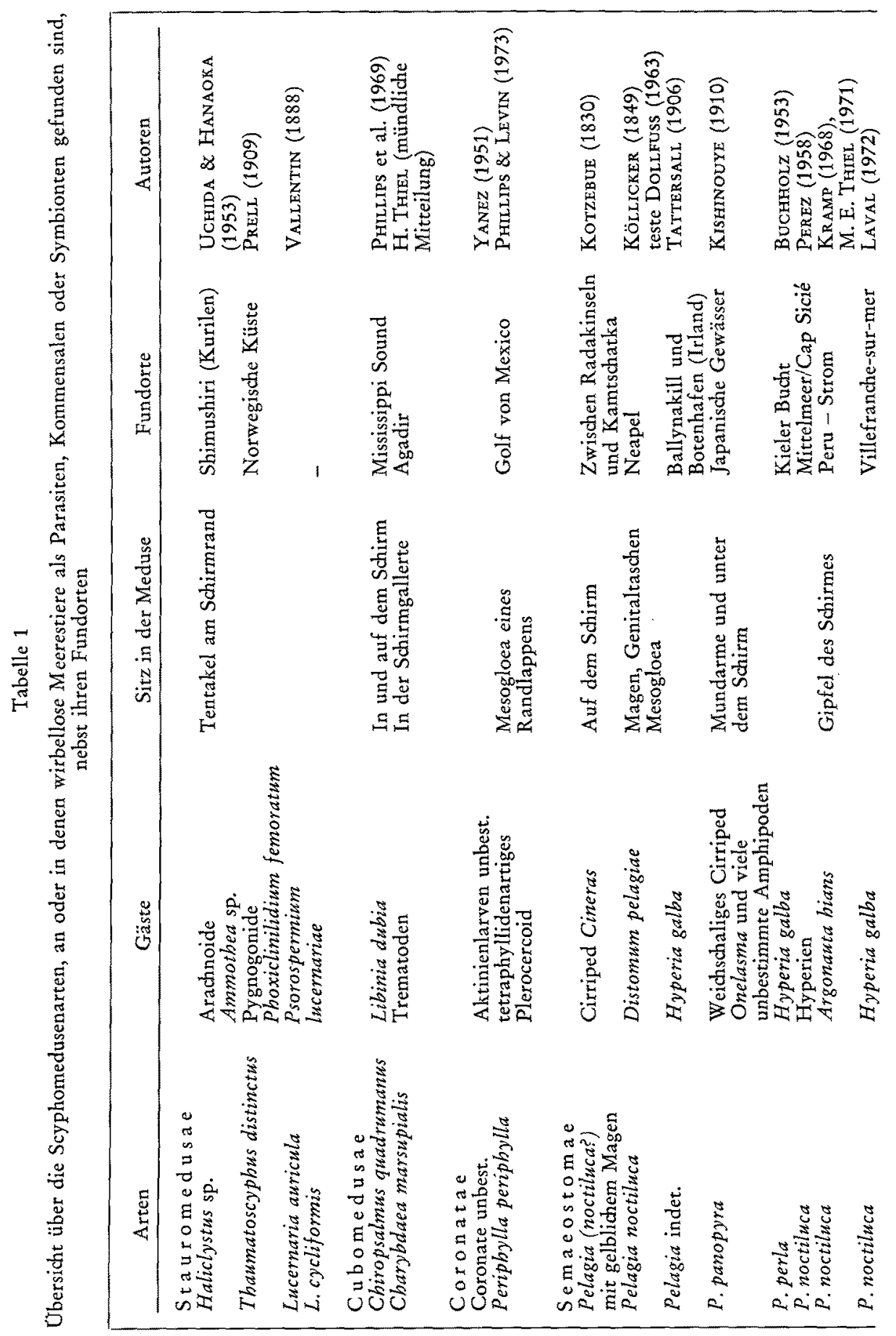



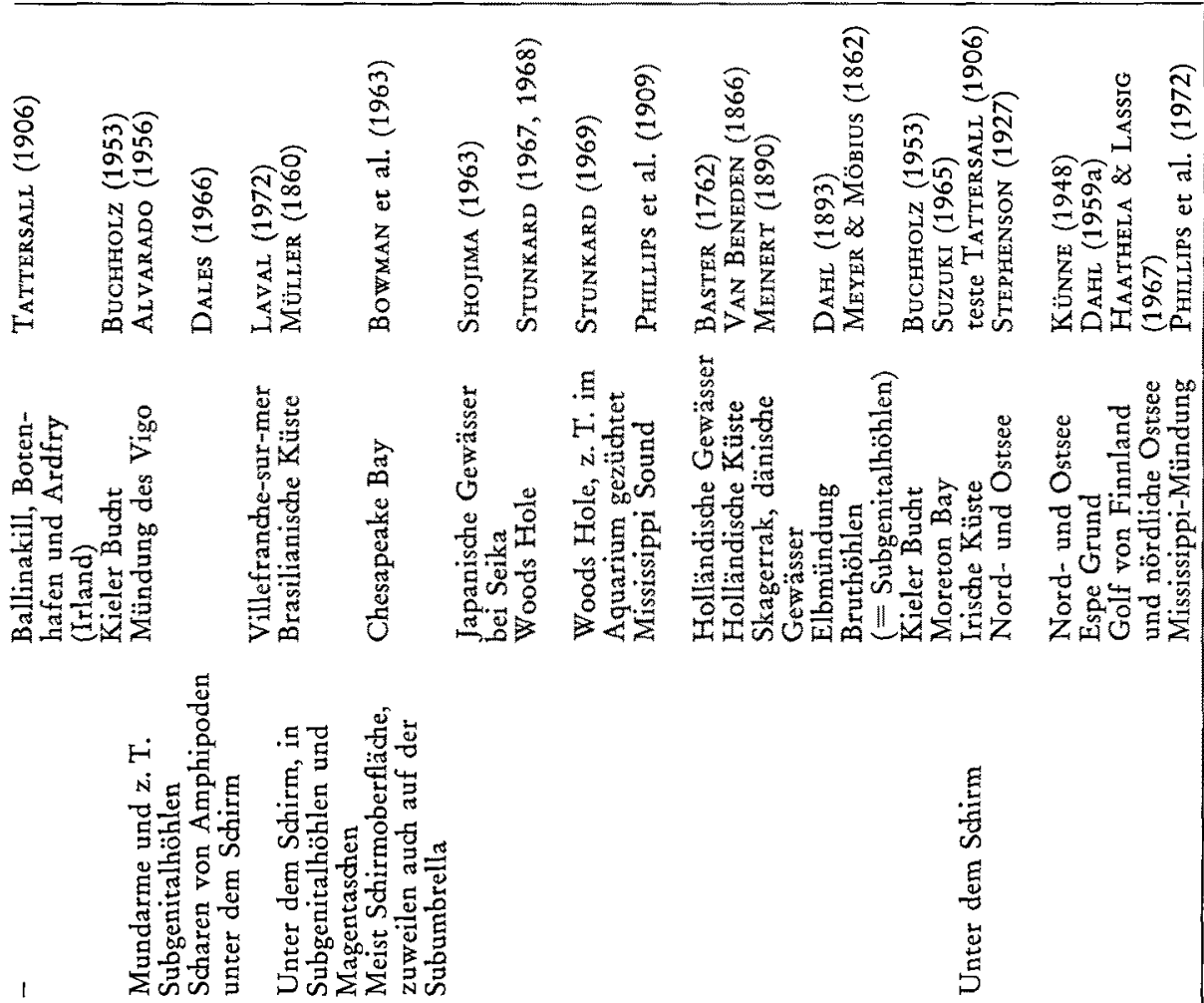

苞

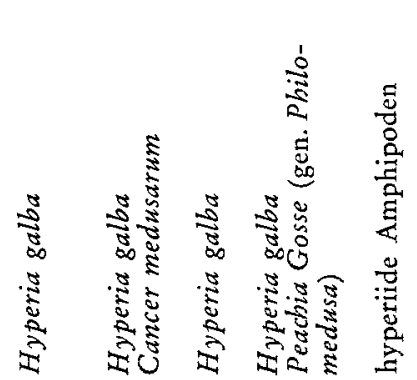

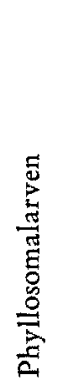

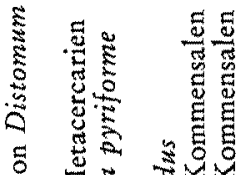
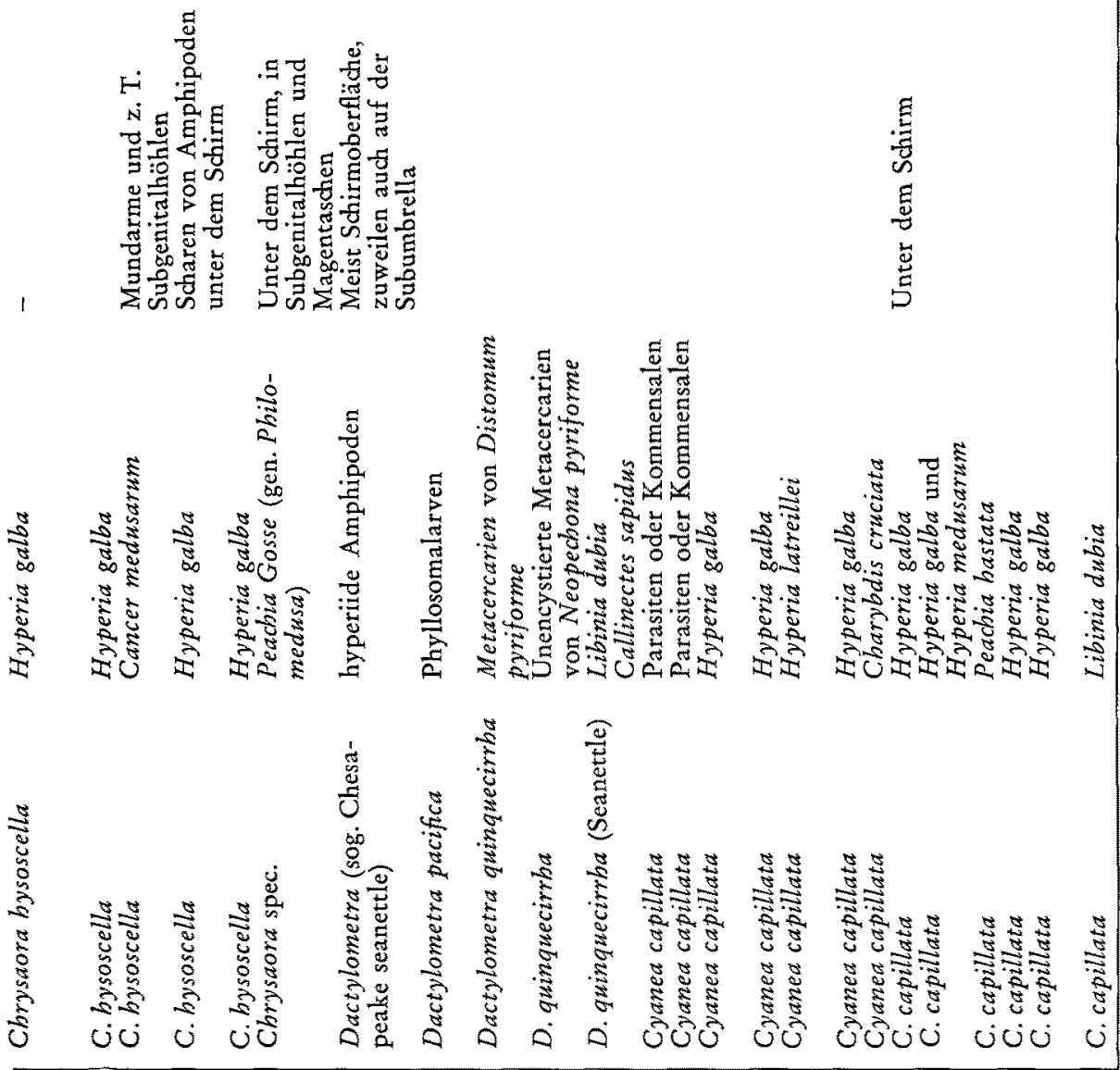


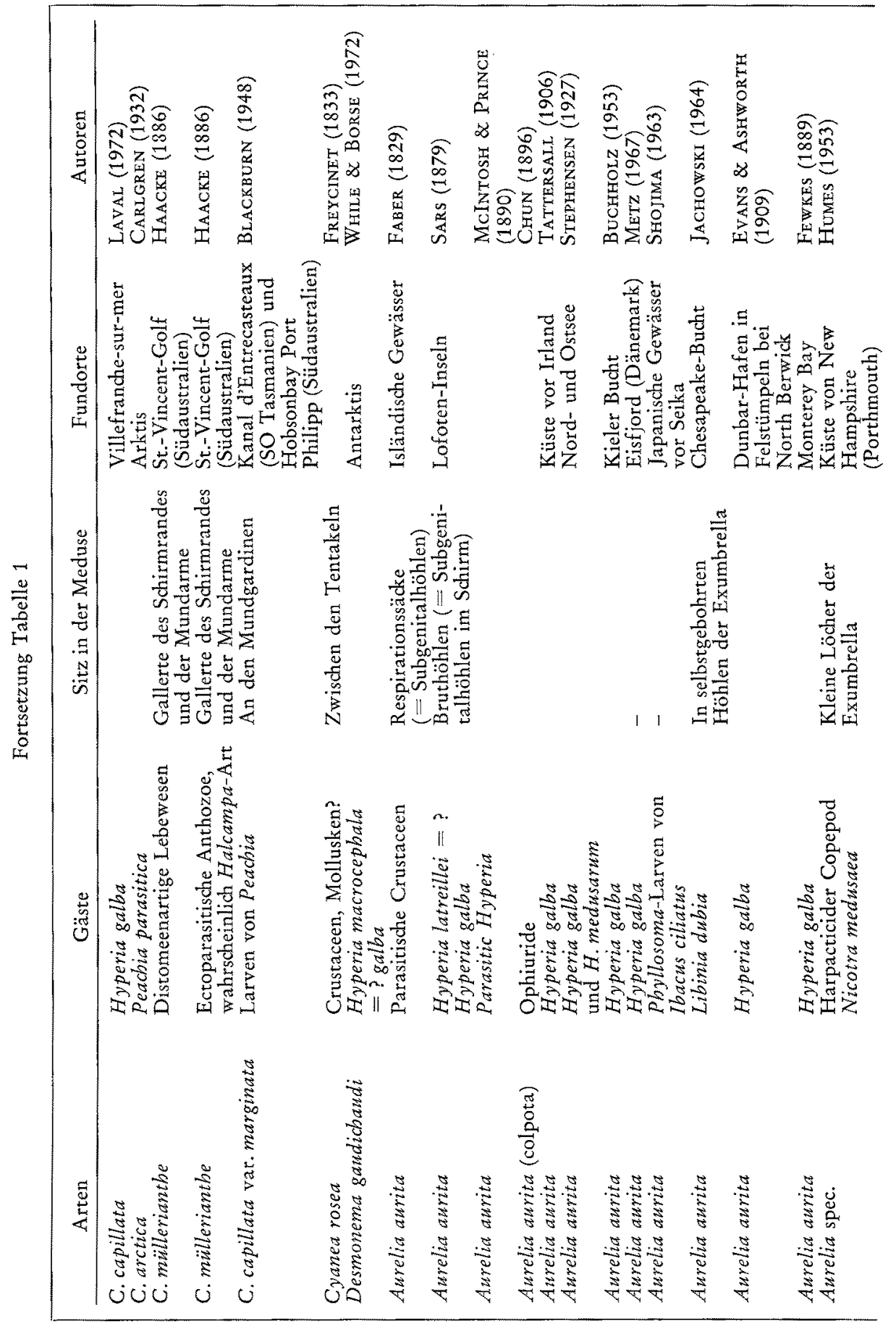




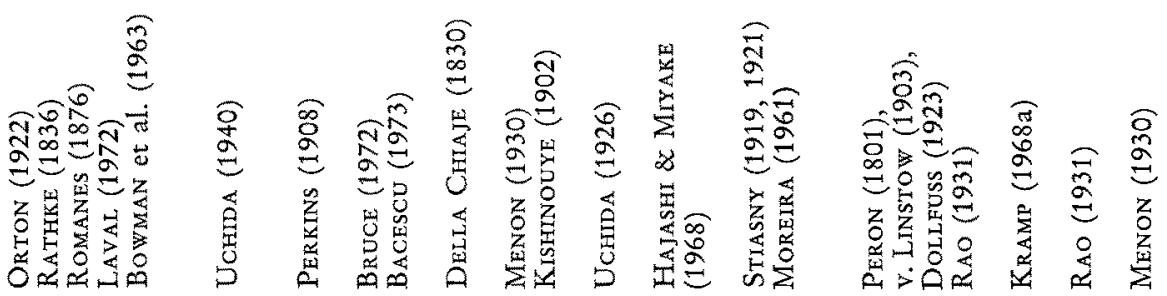

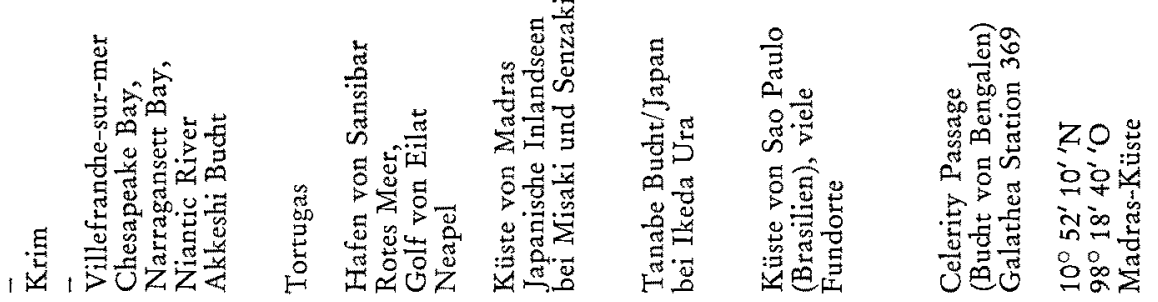
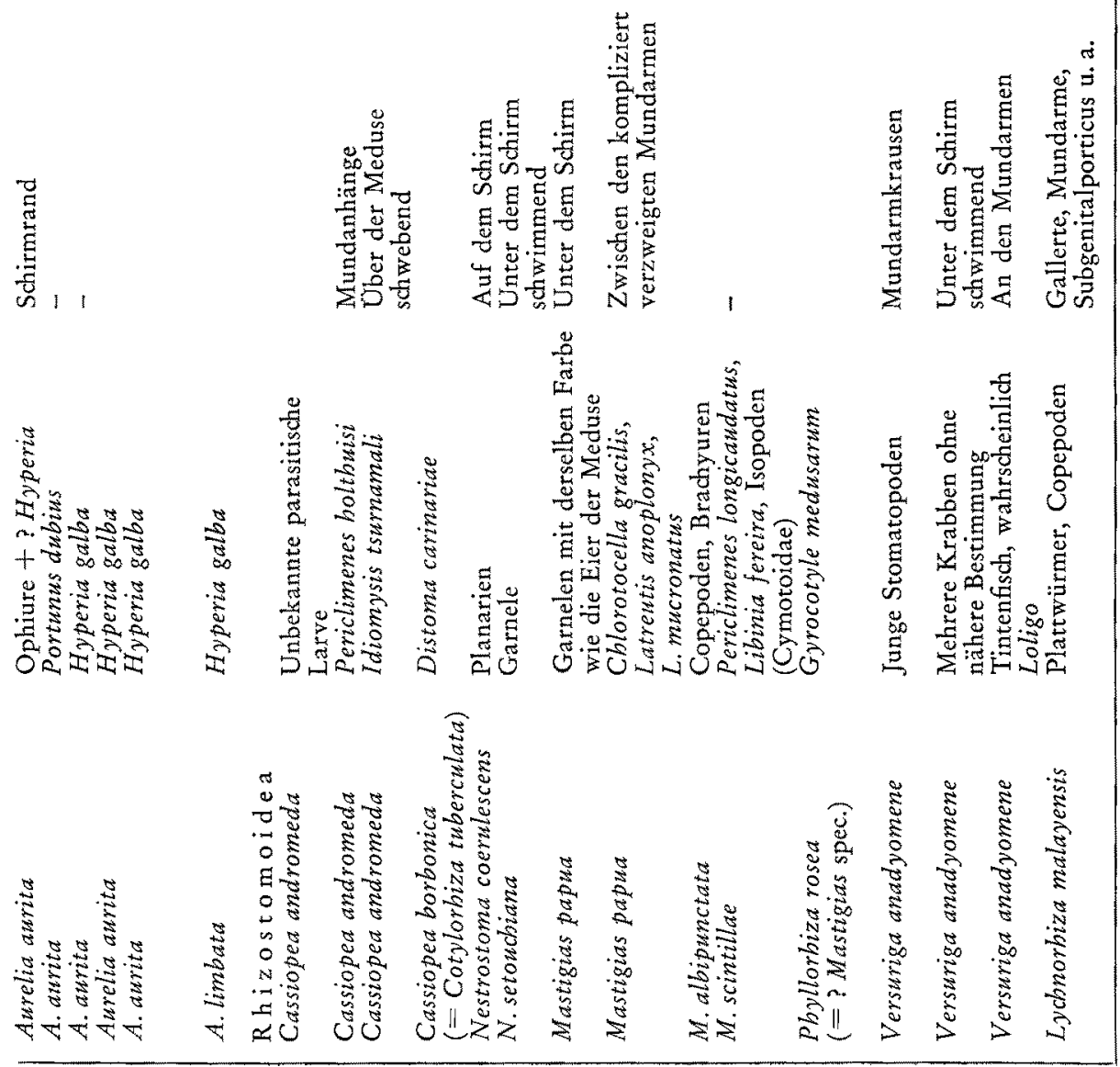


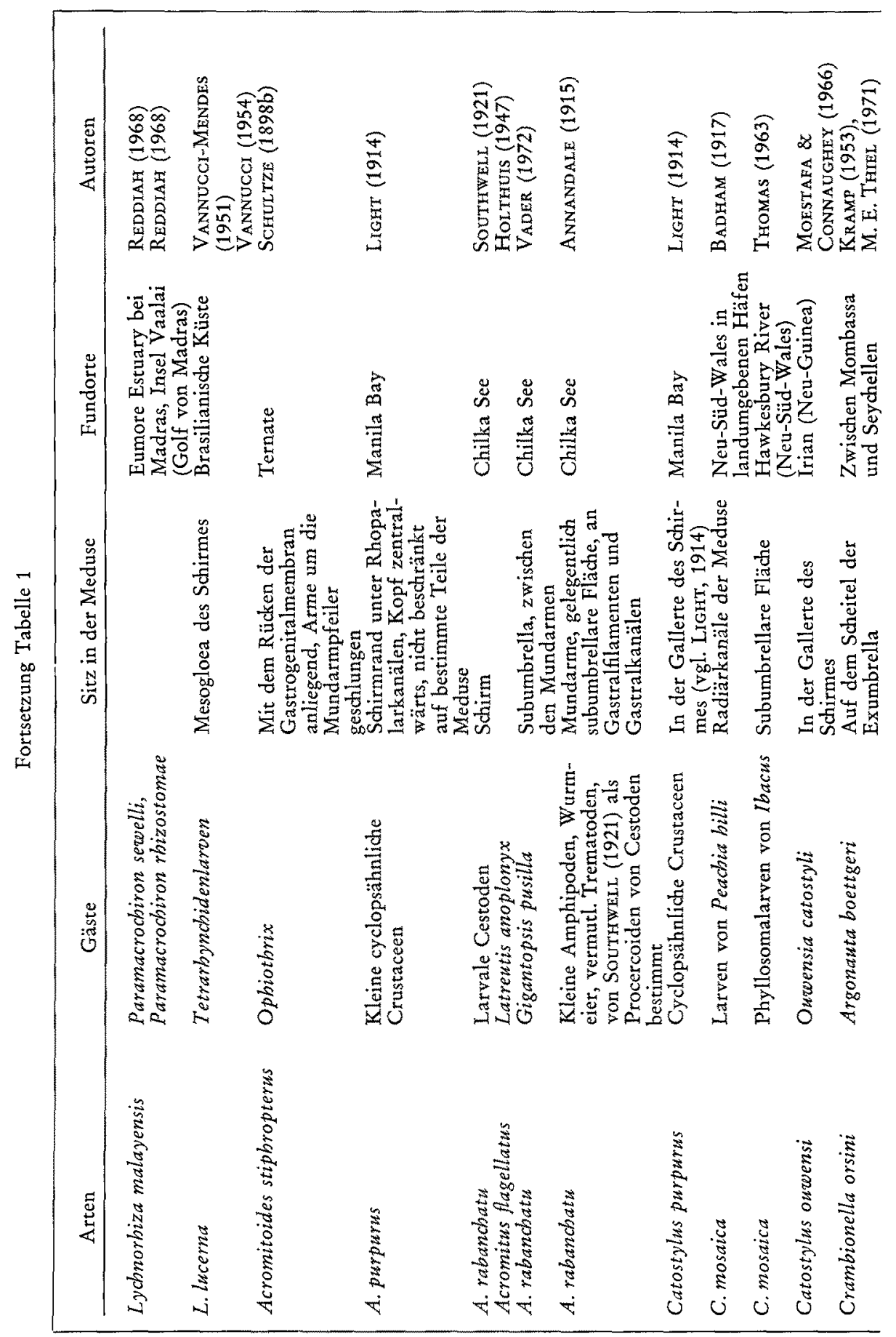




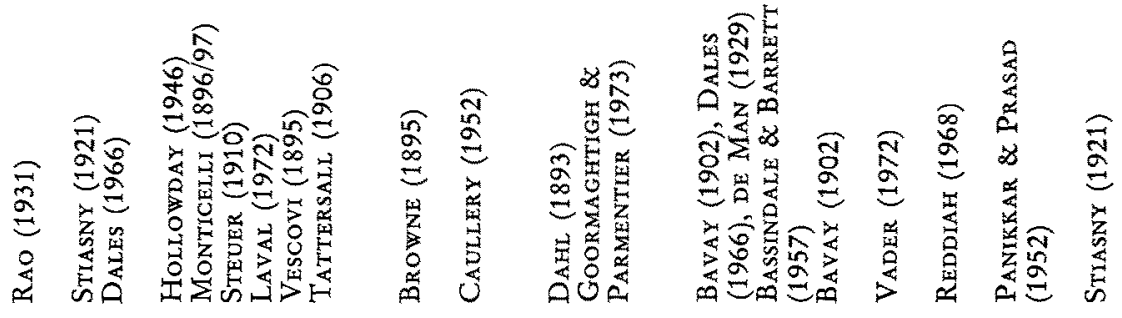

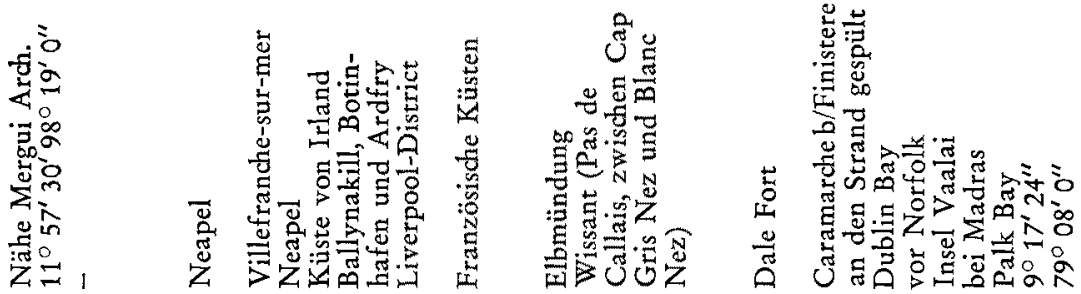

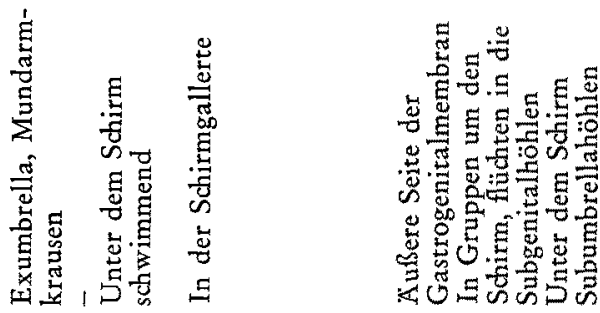

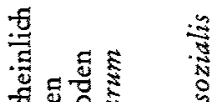

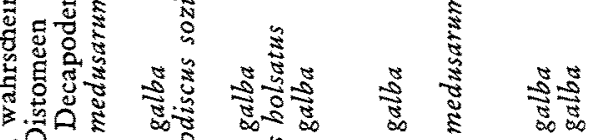

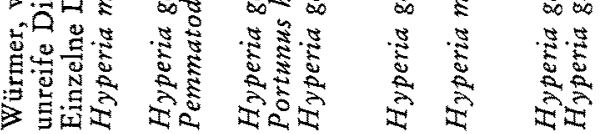

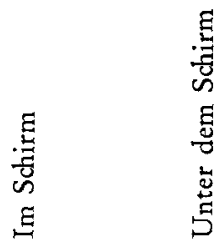

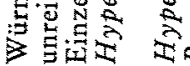

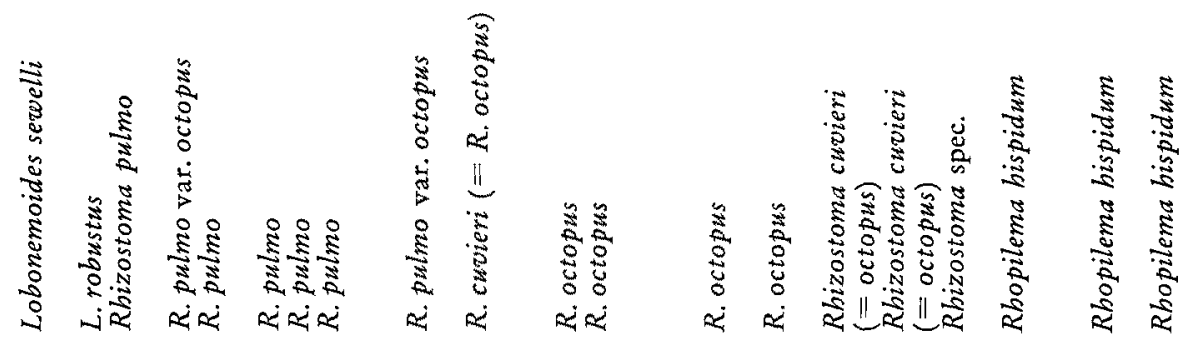




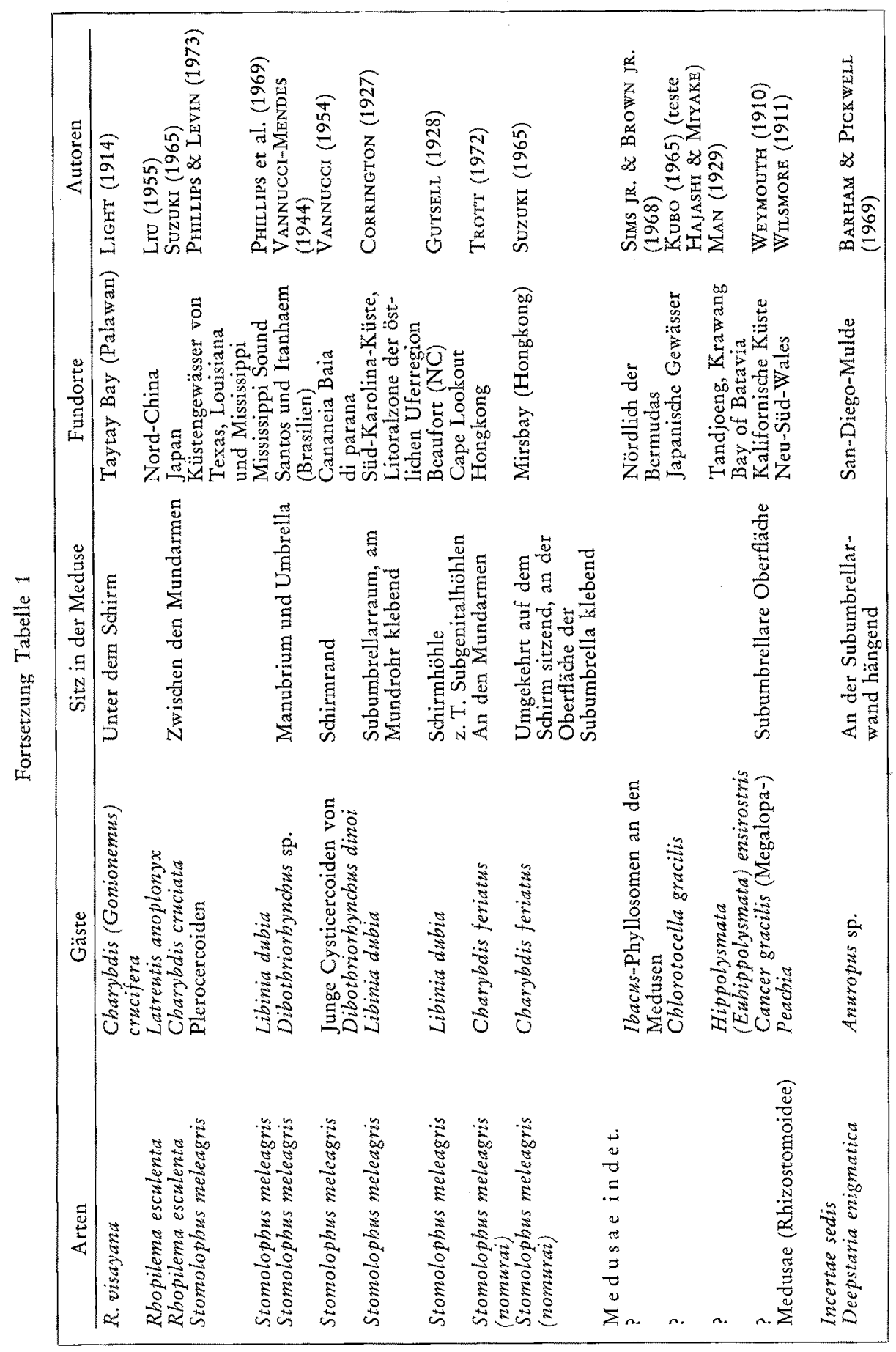


ceen und Cirripedien als Parasiten oder Kommensalen in Arten verschiedener Gattungen der Scyphomedusen festgestellt worden.

In der Folgezeit mehrten sich diese Beobachtungen, wobei z. T. auch bereits die Frage nach der biologischen Bedeutung dieser Vergesellschaftungen gestellt wurde. So beschreibt SARS (1879) zum ersten Male die später bei so vielen Scyphomedusen beobachtete Hyperia galba in Aurelia aurita, und HaAcke (1886) fand in der australischen Cyanea muellerianthe sowohl "Distomeenartige Lebewesen" als auch Aktinienlarven, die fast gleichzeirig auch DENDY (1888) in einer allerdings unbestimmten Meduse in Port Philipp (Südaustralien) beobachtet hat. Um die Jahrhundertwende finden wir dann mehrere Angaben über das Leben von Hyperia galba in unseren heimischen Quallen (McIntosh \& Prince, 1890; Meinert, 1890; Dahl, 1893; Browne, 1895), während Della Valle (1893) dieselbe aus einer allerdings nicht bestimmten Meduse erwähnt und Vescovi (1895) den Fund eines Portunus holsatus in Rbizostoma pulmo beschreibt. Chun (1896) fand bei seiner Bearbeitung der Sammlung Stuhlmann des Hamburgischen Zoologischen Museums einen Ophiuriden in Aurelia colpota von Sansibar, und fast gleichzeitig entdeckte Schultze (1898) in Crambessa (heute Acromitoides) stiphropterus von Ternate ebenfalls einen Ophiuriden. Schließlich stellte MoN'ICELLI in der Gallerte von Rhizostoma pulmo eine bis heute nicht genau bestimmte Form fest, die er Pemmatodiscus sozialis nannte und die Steuer (1910) und Krumbach (1925) als eine Medusengastrula bezeichnet haben.

Schon diese kurze Aufzählung der Funde bis etwa 1900 läßt erkennen, wie weit der Bogen gespannt ist, der die mit Scyphomedusen vergesellschafteten Wirbellosen umfaßt, über die dann im Laufe des 20. Jahrhunderts zahlreiche weitere Beobachtungen angestellt worden sind. Diese sind zusammen mit den friiheren Funden in den Tabellen 1 und 3 zusammengestellt.

\section{UBERSICHT U'BER BISHERIGE BEFUNDE}

Die Angaben über die Scyphomedusen, an und in denen wirbellose Meerestiere als Parasiten, Kommensalen oder Symbionten festgestellt sind, sind in der Literatur weit verstreut und bestehen häufig nur in kurzen Bemerkungen im Text der allgemeinen Beschreibungen der Medusen oder bei den Fundangaben. Sie können daher leicht übersehen werden, so daß die vorliegende Darstellung keinen Anspruch auf Vollständigkeit erheben kann.

Zudem sind die einzelnen in den Medusen gefundenen Arten der Wirbellosen von den Autoren besonders in der ersten Zeit nicht immer mit Gattungs- und Artnamen angegeben worden, sondern mit allgemeinen Bezeichnungen wie z. B. Planarien, Distomeen, Garnelen, Brachyuren und dergleichen oder gar mit ganz unbestimmten Benennungen wie z. B. "Distomeenartige Lebewesen", "Ectoparasiten aus der Klasse der Anthozoen", "Metacercarien von Distomeen", oder "Plerocercoiden von Cestoden" bezeichnet worden, die natürlich in derselben Form wiedergegeben werden mußten. Auch nach der Beschreibung durch die einzelnen Autoren etwa eingetretene klassifikatorische Anderungen der Namen der Arten oder größerer systematischer Einheiten konnten nicht berücksichtigt werden, da in zusammenfassenden Darstellungen z. B. 


\section{Tabelle 2}

Verzeichnis der Scyphomedusenarten, an oder in denen mehrere verschiedene Wirbellose gefunden worden sind

\begin{tabular}{|c|c|c|}
\hline Scyphomedusenarten & Gäste & Autoren \\
\hline \multirow[t]{5}{*}{ Pelagia noctiluca } & Hyperia galba & $\begin{array}{l}\text { Zahlreiche Autoren } \\
\text { (vgl. Tab. 3) }\end{array}$ \\
\hline & Distomum pelagiae & KÖLLIKER (1849) \\
\hline & Metacercarien von Distomum & Dollfuss (1963) \\
\hline & Cirriped der Gattung Cineras & KOTZEBUE $(1830)$ \\
\hline & Argonauta bians. & Kramp (1968), Thiel (1971) \\
\hline \multirow{3}{*}{$\begin{array}{l}\text { P.panopyra } \\
\text { Chrysaora bysoscella }\end{array}$} & Weichschaliges Cirriped Onelasma & KishINOUYE (1902) \\
\hline & Hyperia galba & $\begin{array}{l}\text { TatTersall (1906), BuchHolz } \\
\text { (1953), Dales (1966), Laval } \\
(1972)\end{array}$ \\
\hline & $\begin{array}{l}\text { Cancer medusarum } \\
\text { Philomedusa (= Peachia?) }\end{array}$ & $\begin{array}{l}\text { ALVARADO }(1956) \\
\text { MÜLLER }(1860)\end{array}$ \\
\hline \multirow{3}{*}{$\begin{array}{l}\text { Dactylometra } \\
\text { quinquecirrba }\end{array}$} & Hyperiide Amphipoden & BowMAN et al. (1963) \\
\hline & $\begin{array}{l}\text { Metacercarien von Distomum } \\
\text { pyriforme ( }=\text { Neopechona } \\
\text { pyriforme })\end{array}$ & STUNKARD $(1967,1968,1969)$ \\
\hline & $\begin{array}{l}\text { Spinnenkrabbe (Libinia dubia), } \\
\text { Blaukrabbe (Callinectes sapidus) }\end{array}$ & Phillips et al. (1969) \\
\hline \multirow[t]{4}{*}{ Cyanea capillata } & $\begin{array}{l}\text { Peacbia parasitica } \\
\text { Peachia hastata }\end{array}$ & $\begin{array}{l}\text { CARLGREN (1932) } \\
\text { KüNNE }(1948)\end{array}$ \\
\hline & Hyperia galba & 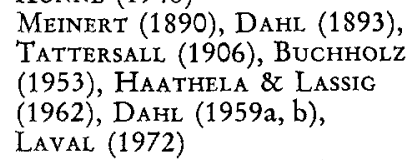 \\
\hline & Hyperia latreillei & MEYER \& MÖBIUS (1862) \\
\hline & Hyperia medusarum & BowMaN et al. (1963) \\
\hline Cyanea muellerianthe & Libinia dubia & PHILlips et al. (1969) \\
\hline Hanea mueverianthe & $\begin{array}{l}\text { Distomeenartige Lebewesen } \\
\text { Anthozoenart (Halcampa?) }\end{array}$ & $\begin{array}{l}\text { HAACKE }(1886) \\
\text { HAACKE }(1886)\end{array}$ \\
\hline \multirow[t]{5}{*}{ Aurelia aurita } & Hyperia galba & $\begin{array}{l}\text { SARS (1879), ROMANES (1876), } \\
\text { TATTERSALL (1906), EVANS \& } \\
\text { AsWORTH (1909), STEPHENSEN } \\
\text { (1927), BUCHHOIZ (1953), } \\
\text { METZ (1967), LAVAL (1972), } \\
\text { BoWMAN et al. (1963) }\end{array}$ \\
\hline & Ophiure & ORTON (1922) \\
\hline & Portutus dubius & RATHKE (1836) \\
\hline & Phyllosomalarven & SHOJIMA (1963) \\
\hline & Libiniat dubia & JACHOWSKI (1964) \\
\hline \multirow[t]{3}{*}{ Cassiopea andromeda } & Unbekannte Larve & Perkins (1908) \\
\hline & Periclimenes boltbuisi & BRUCE (1972) \\
\hline & Idiomysis tsurnamali & BACESCU (1973) \\
\hline \multirow[t]{2}{*}{ Mastigias papua } & Garnelen & UCHIDA (1926) \\
\hline & $\begin{array}{l}\text { Chlorotocella gracilis, Latreutis } \\
\text { anoplonyx, L. mucronata }\end{array}$ & HAYASHI \& MiYaKe (1968) \\
\hline Mastigias scintillae & $\begin{array}{l}\text { Periclimenes longicaudatus } \\
\text { Libinia fereira }\end{array}$ & MOREIRA (1961) \\
\hline Versuriga anadyomene & $\begin{array}{l}\text { Junge Stomatopoden, Tintenfisch } \\
\text { (wahrscheinlich Loligo), mehrere } \\
\text { Krabben }\end{array}$ & $\begin{array}{l}\text { RAO (1931) } \\
\text { KRAMP (1968a) }\end{array}$ \\
\hline
\end{tabular}


Fortsetzung Tabelle 2

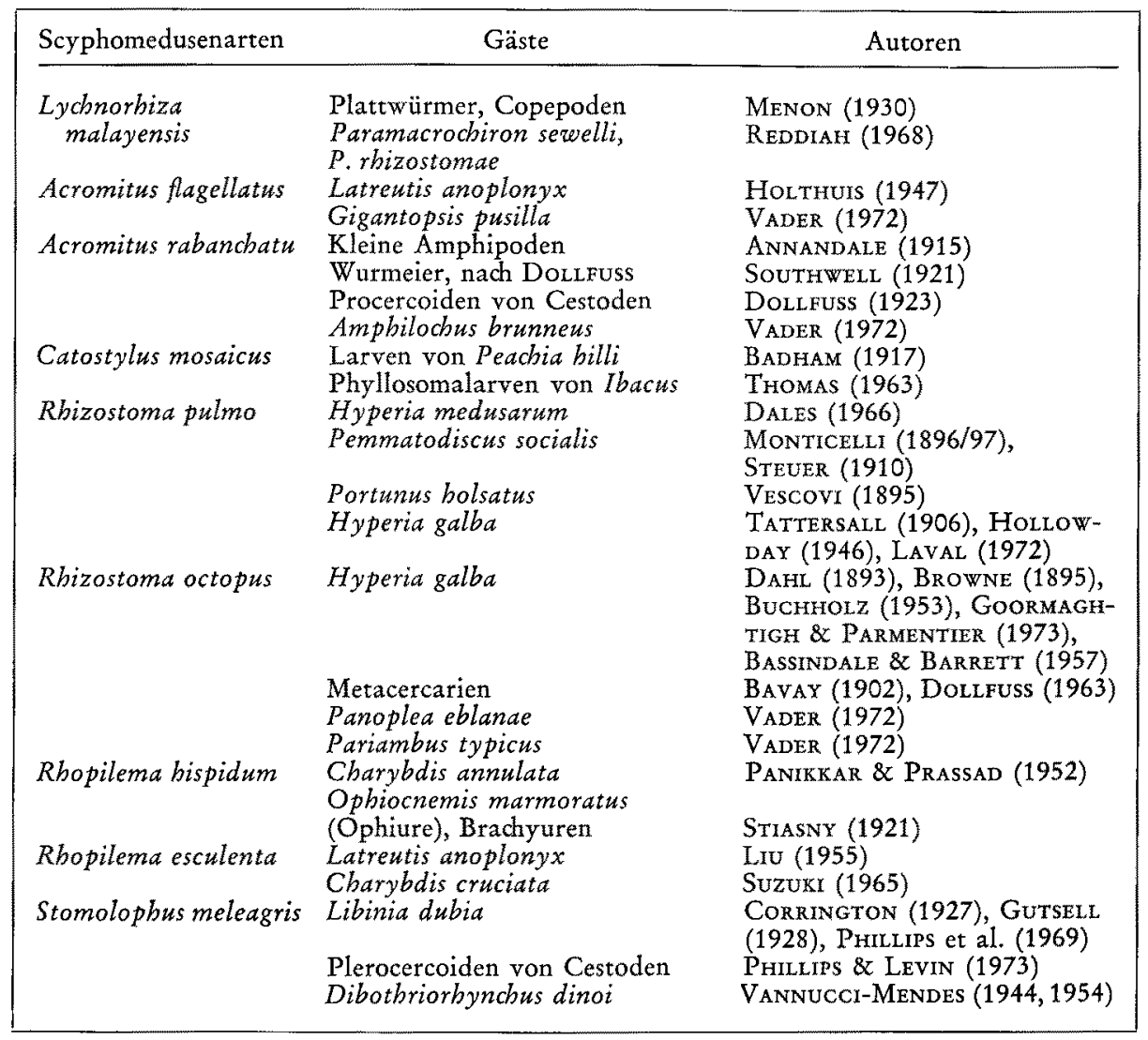

über parasitische Würmer oder über Fische, in denen die Endstadien von in Quallen lebenden Entwicklungsstadien parasitischer Würmer häufig auftreten (z. B. in KükENTHAL: "Handbuch der Zoologie"; Grimpe \& Wagler: "Die Tierwelt der Nord- und Ostsee“; Sprenn. 1938) keine Angaben über Parasiten in Quallen gefunden werden konnten. Erst in neuester Zeit hat VANNUCCI $(1944,1951,1954)$ darauf hingewiesen, daß gewisse Selachier Quallen fressen und die Entwicklungsstadien der Cestoden, die in Quallen leben, dadurch in diese gelangen können.

Zählt man nun an Hand der Tabelle 1 die Arten der Scyphomedusen (ohne die unbestimmten), die mit Wirbellosen vergesellschaftet leben können, so ergibt sich, daß nach der mir bekanntgewordenen Literatur in 51 Arten aus 28 Gattungen der Scyphomedusen Parasiten, Kommensalen oder Symbionten angetroffen wurden. Zugleich läßt die Tabelle erkennen, daß mit ein und derselben Art von Scyphomedusen Wirbellose verschiedener Tierklassen vergesellschaftet sein können. Es besteht also keine enge Bindung zahlreicher Wirbelloser an eine bestimmte Medusenart. Dabei mögen manche Arten nur an eine Quallenart gebunden sein. Das schließt aber nicht aus, daß zugleich 
Tabelle 3

Obersicht über die verschiedenen Tierarten, die in und an Scyphomedusen vorkommen

\begin{tabular}{|c|c|c|}
\hline Taxa & Nachweis in & Autoren \\
\hline $\begin{array}{l}\text { Coelenterata } \\
\text { Aktinienlarven } \\
\text { Peachia, Pbilomedusa } \\
\text { Peachia bastata } \\
\text { Peachia parasitica } \\
\text { Peachia billi } \\
\text { Pemmatodiscus socialis } \\
\text { Peachia } \\
\text { Halcampa spec. } \\
\text { Peachia-Larven }\end{array}$ & $\begin{array}{l}\text { Coronatae (indet.) } \\
\text { Chrysaora spec. } \\
\text { Cyanea capillata } \\
\text { Cyanea arctica } \\
\text { Catostylus mosaica } \\
\text { Rbizostoma pulmo } \\
\text { Rhizostomoidee (indet.) } \\
\text { Cyanea mullerianthe } \\
\text { Cyanea capillata v. marginata }\end{array}$ & $\begin{array}{l}\text { YANEZ }(1951) \\
\text { MÜLLER }(1860) \\
\text { KÜNNE }(1948) \\
\text { CARLGREN }(1932) \\
\text { BADHAM }(1917) \\
\text { MONTICELLI }(1896 / 97) \\
\text { WILSMORE }(1911) \\
\text { HAACKE }(1886) \\
\text { BLACKBURN }(1948)\end{array}$ \\
\hline $\begin{array}{l}\text { Turbellaria } \\
\text { Plattwürmer (ohne nähere } \\
\text { Bestimmung) }\end{array}$ & $\begin{array}{l}\text { Netrostoma coerulescens } \\
\text { Lydhorbiza malayensis }\end{array}$ & Menon (1930) \\
\hline $\begin{array}{l}\text { Trematoda } \\
\text { Distomum pelagiae }\end{array}$ & Pelagia noctiluca & $\begin{array}{l}\text { KöLliKer (1849) } \\
\text { teste DolLfuSS (1963) }\end{array}$ \\
\hline $\begin{array}{l}\text { Trematoden } \\
\text { Metacercarien von Distomum } \\
\text { Metacercarien von Distomum } \\
\quad \text { pyriforme }\end{array}$ & $\begin{array}{l}\text { Cbarybdaea marsupialis } \\
\text { Pelagia noctiluca } \\
\text { Dactylometra quinquecirrba }\end{array}$ & $\begin{array}{l}\text { H. Thiel (mdi. Mitt.) } \\
\text { Dolifuss (1963) } \\
\text { Stunkard }(1967 / 68)\end{array}$ \\
\hline $\begin{array}{l}\text { Unencystierte Metacercarien } \\
\text { von Neopechona pyriforme } \\
\text { Distomumartige Lebewesen } \\
\text { Unreife Distomeen } \\
\text { Metacercarien }\end{array}$ & $\begin{array}{l}\text { Dactylometra quinquecirrba } \\
\text { Cyanea muelleriantbe } \\
\text { Lobonemoides sewelli } \\
\text { Rhizostoma cuvieri } \\
=\text { R. octopus) }\end{array}$ & $\begin{array}{l}\text { HaACKe }(1886) \\
\text { RaO (1931) } \\
\text { BaVAX (1902), Dollfuss } \\
(1963)\end{array}$ \\
\hline $\begin{array}{l}\text { Cestoda } \\
\text { Tetraphyllidenartiges Plero- }\end{array}$ & Peripbylla peripbylla & Phillips \& Levin (1973) \\
\hline Tetrarhynchidenlarven & Lychnorbiza lucerna & $\begin{array}{l}\text { VANNUCCI-MENDES } \\
\text { (1944), VANNUCCI (1951) }\end{array}$ \\
\hline $\begin{array}{l}\text { Plerocercoiden von Cestoden } \\
\text { Plerocercoiden } \\
\text { Dibothriorbynchus dinoi }\end{array}$ & $\begin{array}{l}\text { Acromitus rabanchatu } \\
\text { Stomolopbus meleagris } \\
\text { Stomolopbus meleagris }\end{array}$ & $\begin{array}{l}\text { SouthWEll }(1921) \\
\text { PhILLIPS et al. }(1973) \\
\text { VANNuCCI-Mendes } \\
(1944,1954)\end{array}$ \\
\hline $\begin{array}{l}\text { Nematoda } \\
\text { Gryocotyle medusarum }\end{array}$ & $\begin{array}{l}\text { Pbyllorbiza rosea } \\
(=? \text { Mastigias })\end{array}$ & $\begin{array}{l}\text { PERON (1801), } \\
\text { V. LINSTOW (1903) }\end{array}$ \\
\hline $\begin{array}{l}\text { Crustacea } \\
\text { Parasitische Crustaceen } \\
\text { Harpacticide Copepoden } \\
\text { Nitocra medusarum }\end{array}$ & $\begin{array}{l}\text { Aureliat aurita } \\
\text { Aurelia spec. }\end{array}$ & $\begin{array}{l}\text { FABER }(1829) \\
\text { Humes }(1953)\end{array}$ \\
\hline $\begin{array}{l}\text { Copepoden } \\
\text { Kleine Cyclops-ähnliche } \\
\text { Crustaceen } \\
\text { Paramacrocbiron sewelli, } \\
\text { P. rbizostomae }\end{array}$ & $\begin{array}{l}\text { Mastgias albipunctata } \\
\text { Acromitoides purpurus } \\
\text { (Catostylus purpurus) } \\
\text { Lychnorbiza malayensis }\end{array}$ & $\begin{array}{l}\text { STIASNY (1921) } \\
\text { Light (1914) } \\
\text { REDDiaH (1963) }\end{array}$ \\
\hline $\begin{array}{l}\text { Cirripedia } \\
\text { Cineras spec. } \\
\text { Onelasma spec. }\end{array}$ & $\begin{array}{l}\text { Pelagia noctiluca } \\
P . \text { panopyra }\end{array}$ & $\begin{array}{l}\text { KOTZEERUE }(1830) \\
\text { KISHINOUYE }(1902)\end{array}$ \\
\hline
\end{tabular}


Fortsetzung Tabelle 3

\begin{tabular}{|c|c|c|}
\hline Taxâ. & Nachweis in & Autoren \\
\hline \multicolumn{3}{|l|}{ Amphipoda } \\
\hline $\begin{array}{l}\text { Hyperia galba } \\
\text { Hyperia galba }\end{array}$ & $\begin{array}{l}\text { Pelagia indet. } \\
\text { P. perla }\end{array}$ & $\begin{array}{l}\text { TATTERSALI. (1906) } \\
\text { BuCHHOLZ (1953) }\end{array}$ \\
\hline Hyperia galba & P. noctiluca & LAVAL (1972) \\
\hline Hyperia galba & Cbrysaora bysoscella & TATTERSALL (1906), \\
\hline Hyperia galba & Cbrysaora bysoscella & BucHHOLZ (1953), \\
\hline Hyperia galba & Cbrysaora bysoscella & DaLes (1966), \\
\hline Hyperia galba & Chrysaora bysoscella & LAVAL $(1972)$ \\
\hline Hyperia galba & Cyanea capillata & MeINERT $(1890)$, Dahl \\
\hline Hyperia galba & Cyanea capillata & (1893), TATtersalL \\
\hline Hyperia galba & Cyanea capillata & (1906), STEPHENSEN \\
\hline Hyperia galba & Cyanea capillata & (1927), BuchHolZ \\
\hline Hyperia galba & Cyanea capillata & (1953), DAFI (1959a, b), \\
\hline Hyperia galba & Cyanea capillata & HAATHELA \& LASSIG \\
\hline Hyperia galba & Cyanea capillata & (1967), BoWMAN et al. \\
\hline Hyperia galba & Cyanea capillata & (1963), LAVAL (1972) \\
\hline Hyperia galba & Aurelia awrita & SARS (1879), RomanES \\
\hline Hyperia galba & Aurelia aurita & (1876), Tattersall \\
\hline Hyperia galba & Aurelia aurita & $(1906)$, EVANS \& \\
\hline Hyperia galba & Aurelia aurita & AsHWORTH (1909), \\
\hline Hyperia galba & Aurelia aurita & STEPHENSEN (1927) \\
\hline Hyperia galba & Aurelia aurita & BuchHolz (1953), MeTz \\
\hline Hyperia galba & Anrelia aurita & (1967), LAVAI (1972), \\
\hline Hyperia galba & Aurelia aurita & BoWMAN et al. (1963) \\
\hline Hyperia galba & Rbizostoma pulmo var. octopus & HOLLOWDAX (1946), \\
\hline Hyperia galba & Rbizostoma pulmo & TATTERSALL (1906), \\
\hline Hyperia galba & Rbizostoma pulmo & LAVAL (1972) \\
\hline Hyperia galba & Rhizostoma octopus & DAHL (1893), BROWNE \\
\hline Hyperia galba & Rbizostoma octopus & (1895), BuCHHOLZ \\
\hline Hyperia galba & Rbizostoma octopus & (1953), BASSINDALE \& \\
\hline Hyperia galba & Rhizostoma octopus & BARRETT (1957), \\
\hline Hyperia galba & Rbizostoma octopus & GOORMAGHTIGH \& \\
\hline H. latreillei & Cyanea capillata & $\begin{array}{l}\text { PARMENTIER (1973), } \\
\text { MEYER \& MóBIUS (1862) }\end{array}$ \\
\hline H. medusarum & Cyanea capillata & STEPHENSEN (1927) \\
\hline H. medusarim & Cyanea capillata & Bowman et al. (1963) \\
\hline H. latreillei & Aurelia aurita & MEYER \& MöBUS (1862) \\
\hline H, medusarum & Aurelia asurita & STEPHENSEN $(1927)$ \\
\hline H. medusarum & Rbizostoma pulmo & DaLes (1966) \\
\hline H. medusarum & R.cuvieri ( $=$ octopus $)$ & CAULLLRY (1952) \\
\hline Panoplea eblanae & R. octopus & VADER $(1972)$ \\
\hline Pariambus typicus & R. octopus & VADER $(1972)$ \\
\hline Isopoda & & \\
\hline Anuropus sp. & Deepstaria enigmatica & RusSELL (1967), BARHAM \\
\hline & & \\
\hline Schizopoda & & \\
\hline Idiomysis tsurnamali & Cassiopa andromeda & BACESCU $(1973)$ \\
\hline Stomatopoda & & \\
\hline Stomatopoden (juv, unbest.) & Versuriga anadyomene & RAO (1931) \\
\hline Decapoda & & \\
\hline Garnelen (unbestimmt) & Netrostoma coerulescens & KishinouYe (1902) \\
\hline Garnelen (unbestimmt) & Mastigias papua & UCHIDA (1926a) \\
\hline Garnelen (unbestimmt) & Versuriga anadyomene & Kramp $(1968)$ \\
\hline
\end{tabular}


Fortsetzung Tabelle 3

\begin{tabular}{|c|c|c|}
\hline Taxa & Nachweis in & Autoren \\
\hline Portunus bolsatus & Rbizostoma pulmo & VESCOVI (1895) \\
\hline $\begin{array}{l}\text { Charybdis (Gonionemus) } \\
\text { cruciferd }\end{array}$ & Rhopilema visayana & LIGHT (1914) \\
\hline Charybdis annulata & R. bispidum & $\begin{array}{l}\text { PANIKKAR \& PRASAD } \\
(1952)\end{array}$ \\
\hline Libinia dubia & Stomolopbus meleagris & CORRINGTON (1927), \\
\hline Libinia dubia & Stomolopbus meleagris & Gutsell (1928) \\
\hline Libinia dubia & Chiropsalmus quadrumanus, & Phillips et al. (1969) \\
\hline Libinia dubia & Dactylometra quinquecirrha & \\
\hline Libinia dubia & Cyanea capillata & Phillips (1969) \\
\hline Libinia dubia & Aurelia aurita & JACHOWSKI (1964) \\
\hline L. fereira & Mastigias scintillae & MoReia (1961) \\
\hline Cbarybdis cruciata & Cyanea spec. & Suzuki (1965) \\
\hline Charybdis cruciata & Rhopilema esculenta & Suzuki $(1965)$ \\
\hline C. feriatus & Stomolopbus nomurai & Trott (1972), SUzukI \\
\hline C. feriatus & Stomolophus nomurai & $(1965)$ \\
\hline Chlorotocella gracilis & Mastigias papua & $\begin{array}{l}\text { HAYASHI \& MIYAKE } \\
\text { (1968) }\end{array}$ \\
\hline Latreutis anoplonyx & Mastigias papua & $\begin{array}{l}\text { HAYASHI \& MIYAKE } \\
\text { (1968) }\end{array}$ \\
\hline Latreutis anoplonyx & Acromitus flagellatus & HoLTHuIs (1947) \\
\hline Latreutis anoplonyx & Rhopilema esculenta & LiU $(1955)$ \\
\hline L. mucronatus & Mastigias papua & $\begin{array}{l}\text { HAYASHI \& MIYAKE } \\
\text { (1968) }\end{array}$ \\
\hline Periclimenes longicaudatus & Mastigias scintillae & Moreira (1961) \\
\hline P. holthuisi & Cassiopea andromeda & BRUCE (1972) \\
\hline Phyllosoma-Larven & Dactylometra pacifica, & SHOJIMA (1963) \\
\hline $\begin{array}{l}\text { Phyllosoma-Larven } \\
\text { Phyllosoma-Larven von Ibacus }\end{array}$ & $\begin{array}{l}\text { Aurelia aurita } \\
\text { Catostylus mosaicus }\end{array}$ & Thомаs (1963) \\
\hline $\begin{array}{l}\text { Pygnogonida } \\
\text { Phoxiclinidium femoratum }\end{array}$ & Thaumatoscyphus sp. & PRELL (1909) \\
\hline $\begin{array}{l}\text { Arachnoidea } \\
\text { Ammothea spec. }\end{array}$ & Haliclystus spec. & $\begin{array}{l}\text { UCHIDA \& HANAOKA } \\
\text { (1933) }\end{array}$ \\
\hline \multicolumn{3}{|l|}{ Echinodermata } \\
\hline Ophiotbrix sp. & Acromitoides stipbropterus & SCHultze (1898) \\
\hline Ophiocnemis marmoratus & Rbopilema bispidum & $\begin{array}{l}\text { PANIKKAR \& PRASAD } \\
\text { (1952) }\end{array}$ \\
\hline \multicolumn{3}{|l|}{ Cephalopoda } \\
\hline Loligo spec. & Versuriga anadyomene & RAO (1931) \\
\hline Argonauta bians & Pelagia noctiluca & KRAMP (1968) \\
\hline A. boettgeri & Crambionella orsini & $\begin{array}{l}\text { KRAMP (1953), THIEL } \\
\text { (1971) }\end{array}$ \\
\hline \multicolumn{3}{|l|}{ Incertae sedis } \\
\hline Ouwensia catostyli & Catostylus ouwensi & $\begin{array}{l}\text { MoEstafa \& } \\
\text { ConNAUGHEY (1956) }\end{array}$ \\
\hline
\end{tabular}

in dieser Meduse noch andere Formen oder Arten vorkommen können. Die verschiedenen in den einzelnen Medusenarten gefundenen Gäste sind in der Tabelle 2 zusammengestellt. In Tabelle 3 sind sodann die in den Scyphomedusen gefundenen Gäste nach ihren größeren systematischen Einheiten zusammengestellt worden. Man erkennt daraus, daß weitaus die meisten den Crustaceen angehören, während Coelenteraten 
und Würmer in viel geringerer Zahl, aber auch noch recht häufig vertreten sind und Echinodermen wie Cephalopoden seltene Ausnahmen darstellen. Andererseits sind die letzteren die einzigen größeren Wirbellosen, die in einer engeren Verbindung mit Scyphomedusen gefunden worden sind und daher in der folgenden Erörterung eine gesonderte Darstellung verdienen.

\section{ART UND BIOLOGISCHE BEDEUTUNG DES ZUSAMMENLEBENS VON WIRBELLOSEN MIT SCYPHOMEDUSEN}

Was die biologische Bedeutung dieses Zusammenlebens von Quallen und Wirbellosen angeht, so haben die meisten Autoren diese Frage gar nicht berührt. Vielfach wurde angenommen, daß die Gäste unter dem Schirm der Quallen Schutz suchen, sei es vor verfolgenden Feinden, sei es vor dem hellen Sonnenlicht an der Meeresoberfläche. So bringt noch BerTin (1958) zum Ausdruck, daß Hyperia galba ebenso wie die Fische, die mit Rhizostoma zusammenleben, unter dem Schirm Schutz finden. Auch Dales (1966) äußert sich über Symbiosen bei marinen Organismen wie folgt: „Einige Krebse, die als kommensal betrachtet werden, scheinen bloß Schutz zu suchen, aber viele ziehen wahrscheinlich Nutzen aus der Nahrung ihres Wirtes. Die große Rhizostoma pulmo schützt den Amphipoden Hyperia medusarum MüLIER, von dem man Schwärme unter dem Schirm dieser Meduse schwimmen sehen kann. Ahnlich wird Hyperia galba zusammen mit Rbizostoma octopus und Chrysaora bysoscella gefunden. Es ist möglich, daß durch diese Amphipoden Fische angelockt werden, die dann von den Medusen gefressen werden."

Indessen ist es in vielen Fällen noch eine offene Frage, ob überhaupt bei den Scyphomedusen und ihren Gästen eine echte Vergesellschaftung vorhanden ist. BRUCE (1972) schreibt z. B. in bezug auf die Vergesellschaftung einer pontoniiniden Garnele mit einer leider unbestimmt gelassenen Rhizostomee, daß die Assoziation ziemlich lose und als zufällig anzusehen sei, obwohl die Garnele und auch die Meduse in dem flachen tropischen Wasser in großer Zahl vorhanden seien. In anderen Fällen dürfte wohl wenn parasitische Würmer oder deren Entwicklungsstadien vorliegen -, und das ist hier, wie der folgende Abschnitt zeigt, sehr häufig der Fall - der Schluß berechtigt sein, daß Parasitismus vorliegt. In manchen Fällen lassen auch die von den Autoren angegebenen Fundumstände, z. B. die Art bzw. der Ort, wie und wo der Gast in der Meduse sitzt, Schlüsse auf ihre Beziehung zueinander zu.

\section{Crustaceen}

\section{Hyperia galba}

Die meisten Beobachtungen, die eine Wertung des Verhältnisses zwischen den Wirbellosen und Scyphomedusen zulassen, liegen aus der Klasse der Crustaceen vor. Der wohl am meisten und gelegentlich massenhaft beobachtete Gast mehrerer unserer Medusenarten ist Hyperia galba. BuchHolz (1953) zählte in Aurelia aurita der Kieler 
Bucht bis zu 30 Individuen in einer Meduse. Das scheinbar freie Umherlaufen dieser Krebse unter dem Schirm der Wirte hat schon früh die Frage nach der Art ihrer Beziehung zu den Quallen hervorgerufen. Genauere Untersuchungen darüber sind aber wie auch bei anderen Medusen - erst in neuerer Zeit angestellt worden. So hat DAHL (1959a, b) durch Untersuchung des Darminhaltes von $H$. galba, die in Cyanea capillata gelebt hatte, festgestellt, daß darin nur Nesselkapseln dieser Art vorhanden waren. Er zog daraus wohl mit Recht den Schluß, daß die Hyperien ihre Nahrung - mindestens z. T. - dem Gewebe ihres Wirtes entnehmen und daher als Parasiten anzusehen seien. METZ (1967) stellte über die Lebensumstände von H. galba im Isefjord (Seeland, Dänemark) fest, daß diese Art je nach den Temperaturverhältnissen, wenn Aurelia aurita anwesend ist, fast ganz von den Eiern und Gonaden dieser Meduse lebt, die teilweise völlig ausgefressen wird.

Allerdings ergab sich dabei die Frage, wovon sich diese Amphipoden ernähren, wenn, insbesondere im Winter, keine Medusen vorhanden sind. BuchHolz (1953) nimmt für die Hyperien der Kieler Bucht an, daß sie im Winter auf Hydromedusen wie Melicertum octocostatum, Sarsia tubulosa, Halitholus cirratus und möglicherweise auf die Ctenophore Beroë ovata übergehen. Jedoch besteht da dieselbe Frage, ob diese $\mathrm{Me}$ dusen nicht ebenso wie die Scyphomedusen im Polypenstadium überwintern. KäNDLER $(1950,1961)$ führt sie jedenfalls nicht unter den Hydromedusen an, die den Winter über in der Kieler Förde und dem Fehmarnbelt vorhanden sind. Als solche nennt er nur Rathkea octopunctata (November-Mai) und "die kleine zarte" Obelia (September bis April). Euphysa aurata wurde zwar von August bis Januar gefunden, aber nicht den ganzen Winter hindurch. Ebenso geht aus den Angaben von Broch (1928) und KäNDLER (1961) hervor, daß auch alle übrigen Hydromedusen und unter diesen auch die von BuchHolz angeführten Arten nur bis zum späten Herbst und vom frühen Frühjahr an gefunden werden. Es gibt also bei den einzelnen Arten eine zwar verschieden gelegene, aber doch klar erkennbare Zeit, in der sie im Winter nicht vorhanden sind. Sie können daher das Uberleben von H. galba im Winter nicht sichern.

Dagegen fand KäNDLER durchgehend vom Oktober des einen bis zum Juli des folgenden Jahres Ephyren von Scyphomedusen. Allerdings sind Ephyren mit H. galba als Kommensalen oder Parasiten bisher - soweit mir bekannt - nicht beschrieben worden. Zudem erscheint es fraglich, ob alle diese Arten nicht zu klein sind, um H. galba zu beherbergen und das Uberstehen des Winters zu ermöglichen. Nach Schellenberg (1927) hat das ausgewachsene Männchen von $H$. galba eine Länge von $12 \mathrm{~mm}$, das Weibchen eine solche von $14 \mathrm{~mm}$, während die von BuchHorz (1953) und KäNDLER (1961) genannten Hydromedusen (Tab. 4) bis auf Halitholus cirratus etwa gleich groß oder meist sogar kleiner sind. Eine Aufnahme von $H$. galba kommt also wohl kaum in Frage, zumal die Juvenilen im Frühjahr und Sommer auftreten, wenn genügend große Medusen vorhanden sind. Aus Beobachtungen von H. THIEL (1970) geht schließlich hervor, daß H. galba in den dichten Polstern der Scyphistomae von Aurelia aurita an den Pontons und dgl. des Kieler Hafens und der Kieler Förde leben kann. Der Krebs liegt dabei in Rückenlage zwischen den Polypen, klammert sich mit den hinteren Pereiopoden an diesen fest und frißt die Tentakel von der Spitze her ab. H. galba kann also das pelagische Leben aufgeben und zum Bodenleben übergehen. Das Uberwintern ist damit gesichert, und es darf angenommen werden, daß auch andere bodenlebende 
Tabelle 4

Größe der Hydromedusen, in denen Hyperia galba nach BuchHolz (1953) und KäNDLER (1961) möglicherweise überwintern

\begin{tabular}{|cc|}
\hline Species & Größe \\
\hline Melicertum octocostatum & $10-11 \mathrm{~mm}$ \\
Obelia spec. & $5-6 \mathrm{~mm}$ \\
Sarsia tubulosa & $13 \mathrm{~mm}$ Höhe, $8 \mathrm{~mm}$ Breite \\
Halitbolus cirratus & $16 \mathrm{~mm}$ Höhe, $14 \mathrm{~mm}$ Breite \\
Ratbkea octopunctata & $3 \mathrm{~mm}$ Höhe, $2 \mathrm{~mm}$ Breite \\
Euphysa aurata & $4,5 \mathrm{~mm}$ Höhe, $3,5 \mathrm{~mm}$ Breite \\
\hline
\end{tabular}

Coelenteraten als Nahrung dienen. Gleichzeitig ist durch diese Beobachtungen im Freien und durch diese Experimente gesichert, daß $H$. galba Wirtsgewebe frißt und die Beziehung zu den Polypen von Aurelia aurita und damit auch zu der Meduse als Parasitismus anzusehen ist.

Buchrolz (1953) hat nun weiter festgestellt, daß in der Kieler Bucht außer AureLia aurita und Cyanea capillata auch Chrysaora bysoscella, Rbizostoma octopus und Pelagia perla als Wirte von $H$. galba in Frage kommen. Diese seien allerdings sehr selten. In anderen geographischen Gebieten wie z. B. im Mittelmeer oder in den Tropen kann das indessen ganz anders sein. Laval (1972) fand für das Gebiet von Villefranche sur mer folgende abnehmende Häufigkeit von $H$. galba in den Arten der Scyphomedusen: Rhizostoma pulmo, Aurelia aurita, Cyanea capillata, Chrysaora bysoscella und Pelagia noctiluca. Hier ist also die in der Kieler Bucht nicht vorkommende Rhizostoma pulmo als Hauptwirt für H. galba anzusehen und daher für ihren Bestand bestimmend. Da $R$. pulmo nach GraefFe (1884) im Golf von Triest während des ganzen Jahres vorhanden ist, kann auch $H$. galba ohne Schwierigkeiten das ganze Jahr pelagisch leben. Das gilt indessen nicht für die H. galba in Rhizostoma octopus der holländischen und französischen wie überhaupt der nordwesteuropäischen Küsten, da diese Meduse nach den Beobachtungen von Verwey (1942) und Thiel (1966) etwa von September bis Mitte April dort nicht vorhanden ist.

Wenn Goormathigh \& Parmentier (1973) annehmen, daß H. galba in Rbizostoma octopus der Kanalküste eher parasitisch als kommensal anzusehen sei, weil der Krebs aus der extraintestinalen Vorverdauung dieser Meduse Nutzen ziehe, so würde ihm das natürlich dennoch keine Uberwinterungsmöglichkeit in diesem Gebiet gewähren, abgesehen davon, daß nach THIEL (1964) eine solche Vorverdauung gar nicht bei den Rhizostomeen vorhanden ist.

\section{Andere Crustaceen}

Indessen konnte LAval (1972) an einer anderen Hyperia-Art, H. schizogeneios, experimentell zeigen, daß sich dieser Krebs hauptsächlich von Planktontieren nährt, die von der Meduse gefangen, aber nicht verzehrt werden, sondern zwischen den Trichterkrausen der Mundarme abgelagert sind. Dasselbe gibt VADER (1972) auch für die Caprellide Pariambus typicus an. PanikKar \& Prasad (1952), die unter dem Schirm 
von Rhopilema bispidum die Portuniide Charybdis annulata fanden, lassen die Frage offen, ob der Krebs von den Tentakeln und den Gonaden der Meduse frißt oder sich von den Resten der Quallennahrung miternährt, wie es LAvaI (1972) beschrieben hat.

Bei dieser Art der Ernährung würde der Krebs den Vorteil haben, in der Meduse eine Ansammlung von Nahrung vorzufinden, und andererseits die Medusen den Nutzen, von den zwischen den Trichterkrausen angesammelten, nicht aufgenommenen Nahrungsteilen befreit und somit gereinigt zu werden, was Erbl-Erbesfeld (1955) Putzsymbiose genannt hat und was nach THiel (1970) als Symbiose angesehen werden kann.

Nach Trotт (1972) vermutet auch Suzuki (1965), der in der Mirsbay (Hongkong) Stomolophus nomurai mit Charybdis feriatus und Rhopilema esculenta mit Charybdis cruciata vergesellschaftet fand, daß die Vergesellschaftung der erwachsenen Medusen nur vorïbergehend und die Jungen, die um das Manubrium der Medusen gefunden werden, symbiontisch seien. WEYMOUTH (1910) dagegen nimmt an, daß Cancer gracilis im Megalopa-Stadium in Stomolophus meleagris eindringt und daß diese Verbindung zur Fortentwicklung unerläßlich ist, bis die Krabbe eine Länge von 15-20 mm erreicht hat. Für einen zwangsweisen Kommensalismus ("compulsory commensal life") erklärt BACESCU (1973) auch das Verhalten von Idiomysis tsurnamali zu Cassiopea andromeda im Roten Meer, da es nicht durch gewöhnliches Dredgen festgestellt sei. Genauere Untersuchungen liegen aber darüber nicht vor.

Della Valle (1893) und Pirlót (1932) haben aus der konischen Gestalt der gebündelten Mundarme des in Rbizostoma gefundenen Cirripediers Panoplaea eblanae und einiger anderer Arten von Acanthonotozonatidae auf eine möglicherweise halbparasitische Lebensweise geschlossen. VADER (1972) weist indessen darauf hin, daß nur sehr wenig beweisende Beobachtungen dafür vorliegen. Es sei vielmehr durchaus möglich, daß sich diese Tiere nur zu Ernährungszwecken für kurze Zeit an den Medusen aufhielten. Ebenso bezweifelt er, daß die Caprellide Pariambus typicus, die in großer Zahl an derselben Meduse festgestellt wurde, als echter Parasit angesehen werden kann. Verschiedene Autoren (Dahl, 1959; Bowman et al., 1963; REnshaw, 1965; LAval, 1916, 1968; METZ, 1967) haben das angenommen; LAVAL (1972) hat experimentell gezeigt, daß sich diese Art in der Hauptsache von den planktonischen Tieren ernährt, die von der Meduse gefangen werden.

Einen gewissen Hinweis auf eine ständige Verbindung zwischen den Medusen und den in ihnen lebenden Tieren geben auch die Beobachtungen von LIGHT (1914) und Menon (1930). Ersterer fand, daß die in Rbopilema visayana lebende Charybdis (Gonionemus) crucifera bleicher war, als es für diese Art üblich ist, und sieht darin eine Art von Anpassung an das Zusammenleben mit der Meduse. Dasselbe gilt für die Feststellung Menons (1930), daß Planarien, die auf Netrostoma coerulescens gefunden worden sind, in ihrer Färbung den Farbstreifen auf der Exumbrella dieser Meduse so angepaßt sind, daß sie nur schwer zu erkennen sind.

Bei der Beschreibung des Zusammenlebens der Spinnenkrabbe Libinia dubia mit Stomolophus meleagris erörtert Corrington (1927) unter Hinweis auf die Beobachtungen von WEYMOUTH (1910) über die Beziehungen der echten Krabben (Brachyuren) zu anderen Medusen der Monterey Bay (Californien) verschiedene Möglichkeiten des Zweckes solcher Vergesellschaftung. Eine Erklärung dieser Assoziation geben aber we- 
der er noch Gutsell (1928), de Man (1929), Hayashi \& Mryake (1968) und Bruce (1972). Gutsell neigt zu der Annahme, daß Libinia dubia im späten Larvenstadium unter den Schirm von Stomolophus meleagris vordringt und sich in der Glockenhöhle weiterentwickelt. PHILIpPS et al. (1969) schließen aus ihren Beobachtungen an Dactylometra quinquecirrba im Mississippi-Sund, daß Libinia dubia in diese gelangt, wenn sie sich am oder nahe dem Boden aufhält. Da die Autoren die Krabbe nur in $5 \%$ der Medusen, gleichzeitig aber auch im Freien, gefunden haben, nehmen sie an, daß es sich um eine vorübergehende Vergesellschaftung handelt.

Ganz anders aber ist es bei der von JACHOwsKr (1964) beschriebenen Vergesellschaftung zwischen Aurelia aurita und Libinia dubia in der Mündung des Patuxentflusses (Chesapeake Bay). Libinia dubia wurde zwar mehrfach auf der subumbrellaren Fläche zwischen den Mundarmen gefunden, aber außerdem in von dem Krebs selbst mit Hilfe seiner Chetipeden in die Exumbrella gegrabenen Löchern. Diese Beziehung ist zweifellos als parasitär anzusehen. JACHOwsKI (1964) schreibt, daß bei dem Krebs keinerlei Krankheitserscheinungen (gemeint ist wohl die Wirkung aufgenommener Nesselzellen) aufgetreten seien. Ob das Anfressen des Schirmes bei Aurelia anderer Gebiete oder bei anderen Medusen durch Libinia dubia auch vorkommt, ist bisher nicht bekannt. Daß sie es - soweit wir wissen - bei Stomolophus meleagris nicht tut, könnte daran liegen, daß der Schirm dieser Meduse nicht vorwiegend horizontal gehalten wird, sondern ziemlich senkrecht herabhängt, so daß sich dort kein Plankton ansammeln kann. Sodann ist die Gallerte bei Stomolophus wie bei allen Rhizostomeen härter als bei Aurelia aurita, so daß das Anbohren wohl schwieriger sein dürfte. Schließlich sind bei dieser Meduse ja keine langen Mundarme vorhanden, mit denen der Schirmrand abgewischt werden könnte, und die Nahrungsaufnahme geschieht wie bei allen Rhizostomeen durch die Trichterkrausen. Alle diese Überlegungen machen es verständlich, daß derselbe Krebs in demselben geographischen Gebiet bei diesen beiden Medusen ein so unterschiedliches Verhalten zeigt.

Aber auch Cyanea capillata und Dactylometra quinquecirrba, die nach PHILLIPS et al. (1969) im Gebiet der Mississippimündung ebenfalls von Libinia dubia befallen werden, scheinen nicht in der gleichen Weise angebohrt zu werden, obwohl sie wie Aurelia mehr oder weniger horizontal im Wasser liegen.

Das Einbohren der Libinia dubia in den Schirm der Meduse steht indessen in bemerkenswerter Übereinstimmung mit dem Verhalten von Copepoden, die Humes (1963) auf dem Schirm von Aurelia aurita im Hafen von Porthmouth beobachtet und als Nicotra medusaea beschrieben hat. Ungestört, blieben die Copepoden in taschenförmigen Höhlen in der Exumbrella liegen, deren größte 1-1,5 mm tief und im Durchmesser $1 \mathrm{~mm}$ war. Mehr als 30 solcher Höhlen waren in der Meduse von 3 Zoll Durchmesser, jede mit 10 bis 30 und mehr Copepoden, vorhanden. Wenn diese durch intensive Beleuchtung oder mit einer Nadel gestört wurden, wurden sie aktiv und krochen über die exumbrellare Oberfläche, wobei sie zähe an Bruchstücken der Gallerte klebten. Nach Humes ist nicht klar, ob die Copepoden die Höhlungen selbst erzeugt haben. Er sagt auch nichts darüber, ob die Copepoden sich von der Gallerte ernähren oder von dem auf dem Schirm von Aurelia aurita nach ORTON angesammelten Plankton fressen. Die Tatsache aber, daß sich die Copepoden in den Höhlungen aufhalten, macht es wahrscheinlich, daß eine der beiden Deutungen zutriff. 
Auch Tromas (1963) nimmt an, daß die Phyllosomalarven des Scyllariden Ibacus, die im Hawkesbury-River (New South Wales) von der subumbrellaren Fläche von Catostylus mosaicus abgesammelt worden sind und deren älteste Stadien von demselben Pigment erfüllt waren, das in dieser Meduse vorhanden ist, sich von deren Gewebe ernähren und daher als Parasiten anzusehen sind. Die von SHojima (1963) erwähnte Gewohnheit der Scyllariden-Phyllosoma in den japanischen Gewässern, die Medusen Aurelia aurita und Dactylometra pacifica zu begleiten, läßt zum mindesten auf eine häufige Verbindung schließen. Wenn er weiter schreibr, daß die Medusen, an denen Phyllosomalarven gefunden worden sind, keine intakten Körper und einige von ihnen gar keine Mundarme mehr gehabt hätten, so geht daraus zweifellos hervor, daß hierbei Parasitismus vorliegt.

Dasselbe zeigt der Riesenisopode Anuropus sp., der mit der Tiefseemeduse Deepstaria enigmatica von BARHAM et al. (1969) mit Hilfe eines besonderen Saugapparates auf dem amerikanischen Tauchboot "Deepstar" in $723 \mathrm{~m}$ Tiefe im San Diego Trough in mehreren Exemplaren erbeutet worden ist. Die nähere Untersuchung dieser Medusen (größtes Exemplar $70 \mathrm{~cm}$ Durchmesser) durch Russell (1967) ergab, daß es sich um eine neue Art handelt, deren Zugehörigkeit zu den Semaeostomen oder Rhizostomeen nicht mit Sicherheit entschieden werden konnte. Russell (1967) bezeichnet sie daher nach dem Namen des Tauchbootes "Deepstar" vorläufig mit den neutralen Gattungs- und Artnamen Deepstaria enigmatica (den rätselhaften Tiefenstern). Unter den Wirtstieren ist die Art daher als "incertae sedis" angeführt.

Den Isopoden haben Menzies \& Dow (1958) sowie Barham, Pickwell \& Church (1969) als eine Art der Gattung Anuropus beschrieben, wie sie bis dahin nur einmal in einem Dredgefang der Challenger-Expedition in einem Exemplar von $1 \mathrm{~cm}$ Länge gefunden worden ist. BedDard (1886a, b) hat diese Art als Anuropus branchiatus beschrieben und als eine benthonische Art angesehen. BARHAM et al. (1969) fanden den Riesenisopoden indessen in mehreren Fängen immer zusammen mit Deepstaria enigmatica, jeweils an der Innenwand der Schirmhöhle hängend und im Leben von karmoisinroter Färbung. Aus diesen Beobachtungen und den Funden von zwei Exemplaren von 1,8 und 1,9 cm Länge (das größte Exemplar hatte eine Länge von $8 \mathrm{~cm}$ ) schließen die Autoren, daß diese Anuropide wenigstens einen großen Teil ihres Lebens als "Passagier" von großen Scyphomedusen, vielleicht nur an Deepstaria enigmatica, verbringen. Dabei könnte das Vorhandensein von hakenartigen Thoracalanhängen, spitzen epimeralen Platten, Modifikationen aller Abdominalanhänge und das Fehlen von Augen als Anpassung an ein ständiges Zusammenleben mit der Meduse gedeutet werden. $\mathrm{Da}$ der Isopode offenbar an der Meduse frißt und diese nach Russell (1967) durch die Größe des Gastes in ihrer Schwimmfähigkeit behindert war, wäre das Verhältnis als parasitisch anzusehen.

\section{Das Zusammenleben mit größeren Wirbellosen}

\section{Opbiuriden}

Neben der Beschreibung einer Ophiuride in Aurelia colpota (A. aurita) von Sansibar durch Chun (1896), der keine näheren Angaben über dessen Verhältnis zu der 
Meduse macht, fand Schultze (1898b), daß die in dem Subgenitalsaal von Crambessa stiphroptera ( = heute Acromitoides stiphroptera) eingenistete Ophiotrix dorsal fest an der Gastrovascularwand lag und ihre Arme z. T. um die Armpfeiler der Meduse geschlungen hatte, was auf eine enge Anpassung des Schlangensterns an das Leben in der Meduse schließen läßt. Dabei bleibt allerdings die Frage nach der Art ihrer Verbindung offen. Ebenso ist es bei dem Ophiuriden Ophiocnemis marmoratus, den PanikKar \& Prasad (1952) in der Rhizostomee Rbopilema bispidum entdeckt haben. Ob der Schlangenstern von der Meduse selbst oder von dem Plankton frißt, das von ihr gefangen worden ist, konnte nicht festgestellt werden. Da Detritus die ursprüngliche Nahrung des Ophiuriden sein dürfte, erscheint es wahrscheinlich, daß die Beziehung dieser beiden Organismen zueinander als Nahrungsparasitismus gewertet werden muß. Als Symbiose (Putzsymbiose nach Eibl-Eibesfeld , 1955) müßte das Verhältnis dann angesehen werden, wenn der Schlangenstern die Trichterkrausen der Rhizostomee von Nahrungsresten befreit, die zwischen den Trichterkrausen zurückgeblieben sind und von der Meduse nicht mehr aufgenommen werden konnten (THIEL, 1970).

\section{Cephalopoden}

Das Zusammenleben von zwei Arten des Papiernautilus, Argonauta boettgeri und A. hians, mit den Scyphomedusen Crambionella orsini bzw. Pelagia noctiluca ist zuerst von Kramp (1956) auf der Galathea-Expedition im Indischen Ozean beobachtet und später (1968) auch aus dem Perustrom auf Grund amerikanischen Materials beschrieben worden. Er beschreibt diese Beobachtung der um das Schiff herumschwimmenden Medusen mit den darauf "reitenden " Tintenfischen (ThIEL, 1971) zwar als ein wunderbares Schauspiel, sagt aber sonst weiter nichts Näheres über die Art und Bedeutung dieses Zusammenlebens. THIEL (1971) weist indessen darauf hin, daß diese Assoziation an zwei geographisch so weit voneinander entfernten Orten wie im Indischen Ozean bei Madagaskar und im Perustrom vor der chilenischen Küste und bei systematisch so weit entfernt stehenden Medusen wie der Rhizostomee Crambionella orsini und der Semaeostome Pelagia noctiluca wohl kaum als einmalig oder zufällig angesehen werden könne, sondern einem biologischen Bedürfnis entsprechen dürfte. Wenn nun Kramp (1953) bei der Beschreibung dieser Beobachtung des Zusammenlebens den Ausdruck "ensconce" (= es sich bequem machen) anwendet, so kann man darin eine gewisse Deutung desselben sehen, die etwa einem Ausnutzen des Substrates oder einem Ausruhen auf demselben entspräche, wie es bei vielen pelagischen Organismen beobachtet worden ist, was aber hier noch genauerer Untersuchungen bedarf.

\section{Coelenteraten, Turbellarien, Trematoden, Cestoden und Nematoden}

Während bei den Crustaceen und den Ophiuriden das Verhältnis zu den Medusen nicht immer sicher eingeschätzt werden kann, ist die Beziehung für solche Tierformen, die in der Gallerte oder in dem Gastrovascularsystem der Scyphomedusen leben oder 
als Parasiten und deren Entwicklungsstadien bekannt sind, wohl eindeutig als Parasitismus zu erkennen. Das ist daher bei der Auffindung von Distomeen in Rhizostoma cuvieri ( $R$. octopus) durch BAvay (1892), in Lychnorbiza malayensis durch MenoN (1930) und in Lobonemoides sewelli durch Rao (1931) der Fall. Auch die von v. LinsTOw (1903) beschriebene Gyrocotyle medusarum in der allerdings von ihm selbst mit Fragezeichen versehenen Phyllorbiza rosea von PÉRON \& Lesueuer (1801) ist wohl als Parasit dieser Meduse anzusehen.

Am besten bekannt und am sichersten als Parasitismus zu betrachten sind die von Badham (1917), Dollfuss (1929), Vannucci-Mende (1944) und Moestafa \& McConnaughey (1966) beschriebenen Fälle. Badram (1917) beobachtete vor der Küste Südaustraliens in Crambessa (= Catostylus) mosaica die Larve von Peachia billi. Dabei stellte er fest, daß die Larve, die in den Radiärkanälen von Catostylus mosaicus lebt, ständig von einem Strom der Gastrovascularflüssigkeit umflossen wird, die Aktinienlarve also im Nahrungsbrei der Meduse und damit wohl als Parasit lebt. Weiter beobachtete BADHAM (1917), daß die Larven, die er in der Meduse fand, von 3-40 mm heranwachsen, sich ein Loch in die Subumbrellarwand bohren und durch dieses ins Freie gelangen. Er konnte diesen Vorgang selbst verfolgen, indem er sowohl Larven in den Radiärkanälen in der Nähe des Loches, als auch andere, die das Loch, mit ihrem oesophagealen Ende heraustretend, ausfüllten und schließlich die freien Larven an den Mundarmen beobachtete. Die aus der Meduse hervorkommenden jungen Tiere stimmten in ihrem anatomischen Bau mit den Erwachsenen von Peachia billi überein, die im Freien leben und von WILSMORE (1911) ebenfalls aus einer Bucht der südaustralischen Küste beschrieben worden sind.

Ebenso kann das Vorkommen von Entwicklungsstadien von Cestoden und Trematoden in Quallen wohl sicher als parasitisch angesehen werden. So erkannte SoutHwELL (1921), daß die von Annandale (1915) in Acromitus flagellatus des Chilkasees gefundenen und von ihm vorläufig als Eier angesehenen kugeligen Gebilde Plerocercoide eines Cestoden darstellen. Diese Beobachtung wurde durch die Beschreibung von Tetrarhynchidenlarven in Stomolophus meleagris und Lychnorbiza lucerna durch VANNUcciMendes (1944) und Vannuccr (1951) bestätigt. Vannucci-Mendes (1944) fand in Stomolophus meleagris zwei Typen von Larven, eine von zylindrischer und eine von rundlicher Gestalt. Der erste Typ geht in den zweiten über, der ein schwanzloses Cysticercoid bildet. Es besitzt vorn ein ausstülpbares Organ, das VanNucci-Mendes als Protoscolex bezeichnet und das mit kleinen dachziegelartig angeordneten Haken versehen ist. Bei der lebenden Larve wird dieses Organ beständig vorgestreckt und zurückgezogen. VannuCCI-Mendes stellt $z$ war ausdrücklich fest, daß es sich nicht um Procercoide handele, wie sie SouthсотT im Indischen Ozean in Acromitus flagellatus beobachtet habe, aber es ist doch wohl anzunehmen, daß es sich um entsprechende Gebilde handelt. VANNUCCI-MENDES (1944) kommt auf Grund ihrer genauen anatomischen Untersuchungen sodann zu dem Schluß, daß es die Larven einer neuen Art der Gattung Dibotbriorbynchus sein müssen. Schließlich stellt sie fest, daß der Riesenhai, der Katzenhai und zahlreiche Teleosteer als quallenfressend bekannt seien, so daß die Larven von Dibotbriorbynchus in den genannten Medusen einen $Z$ wischenwirt haben könnten.

Als Parasiten bezeichnen Moestafa \& Connaughey (1966) auch den von ihnen in der neu entdeckten Meduse Catostylus ouwensi gefundenen und als neue Art, Ouwensia 
catostyli, beschriebenen Wurm, dessen systematische Zugehörigkeit allerdings weder von ihnen selbst noch von auswärtigen Spezialisten ermittelt werden konnte. Die Würmer lagen in kleinen Kanälen in der Gallerte der Meduse dicht unter der subumbrellaren Muskulatur entlang des Schirmrandes, in dem Subgenitalporticus und im oberen Teil der Mundarme ihres Wirtes. Thre Länge betrug $0,2 \mathrm{~mm}$ bei den kleinsten und über $3 \mathrm{~mm}$ bei den größten Exemplaren. Die Abbildungen des ganzen Wurmes in der Gallerte liegend und von Schnitten durch Rumpf, Vorder- und Hinterende zeigen ein ziemlich hochentwickeltes Tier mit kreuzförmigem Mund, dem sog. "Auge", das wohl eher einen Ocellus darstellt, Nervenmasse und cuticularen Zähnchen. Das Vorhandensein eines "Auges", das allerdings nicht bei allen Individuen festgestellt werden konnte, und der cuticularen Zähnchen lassen vermuten, daß es sich um das - vielleicht letzte Stadium einer parasitischen Larve handelt, deren Adulte im Freien leben, da sonst weder das "Auge" noch die Zähnchen nötig wären. Darauf deutet auch die Beobachtung der Autoren hin, daß die großen Exemplare mit ihrem Vorderende durch einen kleinen Porus der Gallerte herausragen, durch den der Wurm ins Freie gelangen könnte.

In ihrer Schrift über die Cestodenlarven in Scyphomedusen aus dem Golf von Mexiko stellen Phillips \& Levin (1973) das Vorderende von Ouwensia catostyli in Vergleich mit den von ihnen in Stomolophus meleagris gefundenen Plerocercoiden, mit denen es in der Tat große Ähnlichkeit hat. Danach würde O. catostyli als ein Entwicklungsstadium eines Cestoden anzusehen sein. Andererseits erinnert die ganze Lage und Anordnung der cuticularen Zähnchenreihen auf der Oberfläche des Schlundes sehr an eine Schneckenradula. Es liegt daher der Gedanke nahe, daß es sich bei diesem "Wurm" auch um ein Entwicklungsstadium einer Schnecke handeln könnte.

Eine weitere Cestodenlarve fanden Phillips \& Levin (1973) in der Mesogloea eines Randlappens der coronaten Meduse Periphylla periphylla aus dem Golf von Mexiko. Die Larve stellt ein tetraphyllidenartiges Plerocercoid dar, hat eine Länge von $2 \mathrm{~mm}$ und ist relativ undifferenziert. $\mathrm{Da}$ ein ähnliches Plerocercoid im Darm von Tiefseefischen der Familie Macrouridae im Golf von Mexiko vorkommt, besteht nach Phillips \& Levin die Möglichkeit, daß Fische der Genera Caranx, Peprilus, Poronotus und Chloroscombrus, die vielfach mit der Qualle Stomolophus meleagris vergesellschaftet sind (THIEL, 1970), zum vorübergehenden oder endgültigen Wirt für das Plerocercoid von S. meleagris werden. S. meleagris dient einer großen Zahl von Teleosteern und Crustaceen als Nahrung, womit der parasitische Lebenskreis geschlossen wäre.

Parasitismus liegt sicher auch bei den in Dactylometra quinquecirrba beobachteten Metacercarien des digenetischen Trematoden Neopechona pyriforme vor, deren Vorhandensein und weitere Entwicklung von STUNKARD (1967, 1968, 1969) beschrieben und die von ihm bis zum ausgewachsenen Tier in Aquarien geziuchtet worden sind. Um zunächst die Identität der Arten zu prïfen, züchtete STUNKaRd die Metacercarien in zwei Hydromedusen und der Scyphomeduse Dactylometra quinquecirrha. Er konnte feststellen, daß sie mit den in der Natur bei Fischen gefundenen identisch waren. Dann wurden die experimentell mit den Metacercarien infizierten Medusen in Aquarien zu 3 Fischen der Art Stenotoma chrysops gesetzt. Vier Wochen später wurden die Fische untersucht. Dabei fand Stunkard 47, 24 bzw. 20 Individuen der Ausgangsart Neopechona pyriforme. Die Quallen mit den Metacercarien müssen demnach von den 
Fischen gefressen worden sein und die Metacercarien sich in diesen zu den voll ausgebildeten Trematoden weiterentwickelt haben.

\section{ZUSAMMENFASSUNG}

1. Das Vorkommen wirbelloser Tiere als Parasiten, Kommensalen oder Symbionten in Scyphomedusen, das zwar in zahlreichen Schriften über diese Medusen erwähnt, aber nicht in größere Fachwerke aufgenommen und daher wenig bekannt ist, wird an Hand der Literatur der Scyphomedusen zusammenfassend dargestellt.

2. In Tabellen sind einerseits die Medusen mit den an ihnen beobachteten Wirbellosen zusammengestellt und andererseits die einzelnen Arten der Wirbellosen mit den $\mathrm{Me}$ dusen, in denen sie gefunden sind, nach ihren systematischen Gruppen aufgeführt.

3. Es ergibt sich daraus, daß 51 Arten aus 28 Gattungen der Scyphomedusen Wirbellose als Parasiten, Kommensalen oder Symbionten enthalten, die den Actiniariae, Turbellariae, Trematoda, Cestoda, Nematoda und verschiedenen Gruppen niederer und höherer Crustaceen sowie sogar den Echinodermen und Cephalopoden angehören können, wobei allerdings Crustaceen bei weitem am häufigsten sind und in ein und derselben Meduse mehrere verschiedene Wirbellose, z. T. gleichzeitig, vorkommen können.

4. Die Frage, welcher Art diese Vergesellschaftung der Wirbellosen mit den Medusen ist, d. h. ob Parasitismus, Kommensalismus oder Symbiose vorliegt, wird für die einzelnen Fälle erörtert; vielfach mußte diese ungeklärt bleiben.

5. Beweise für eine der drei möglichen Beziehungen fanden sich bei den Crustaceen nur für Parasitismus. Bei den Coelenteraten, Turbellarien, Trematoden, Cestoden und Nematoden kann meist das Verhältnis mit Sicherheit als Parasitismus, verbunden mit einem Generationswechsel, angesehen werden, wobei die Medusen die Zwischenwirte für die Entwicklungsstadien darstellen, deren Adulte in Fischen leben, welche die Medusen fressen. Als Putzsymbiose kann das Zusammenleben der Rhizostomee Rhopilema hispidum mit dem Ophiuriden Ophiocnemis marmoratus betrachtet werden.

6. Die Umwandlung von Metacercarien des Trematoden Neopechona pyriforme aus Dactylometra quinquecirrba in dem Fisch Stenostoma zu dem Adultus, die experimentell nachvollzogen worden ist, wird beschrieben.

7. Die überraschenden Funde eines Riesenisopoden Anuropus spec. von $7 \mathrm{~cm}$ Länge in der neu aufgefundenen Tiefseemeduse Deepstaria enigmatica (bis zu $70 \mathrm{~cm}$ Durchmesser) sowie des Parasiten Ouwensia catostyli in der Meduse Catostylus ouwensi werden näher beschrieben und diskutiert.

8. Das "Reiten" von zwei Arten des Papiernautilus, Argonauta boettgeri und A. hians, auf den Medusen Crambionella orsini und Pelagia noctiluca wird als ein Ausruhen auf dem Substrat verstanden. 


\section{ZITIERTE LITERATUR}

Alvarado, R., 1956. El "cangrejito" de las medusas. Boln. R. Soc. esp. Hist. nat, 53, 219-220.

Annandate, N., 1915. Fauna of the Chilka Lake. The coelenterates of the lake with an account of actiniarian of brackish waters in the Gangetic delta. Mem. Indian Mus. Calcutta 5, 65-114.

Bacescu, M., 1973. A new case of commensalism in the Red Sea: the mysid Idiomysis tsurnamali n. sp. with the Coelenterata Megalactis and Cassiopea. Revue roum. Biol. (Zool.) 16 (1), 3-7.

BadHam, C., 1917. On a larval actinian parasitic in a Rbizostoma. Q. Jl microsc. Sci. 62, 221-230.

Barham, E. G. \& Pickwell, G. V., 1969. The giant isopod, Anuropus, a scyphomedusan symbiont. Deep-Sea Res. 16, 525-529.

Bassindare, R. \& Barrett, J. H., 1957. The Dale Fort marine fauna. Proc. Bristol Nat. Soc. $29,227-328$.

BASTER, J., 1762. Natururkundige Uitspanningen behelzende sommige waarnemingen over sommige Zeeplanten en Zeeinsekten. Haarlem.

Bavay, A., 1962. Note sur un distome parasite d'une méduse. Archs. Parasit. 53, 199-200.

BEDdard, F. E., 1886a. Preliminary notice of the Isopoda collected during the voyage of HMS Challenger pt. III. Proc. zool. Soc. Lond. 1886 (1), 97-122.

- 1886b. Report on the Isopoda. Rep. scient. Results Voyage HMS Challenger (Zool.) 17, $1-178$.

Beneden, J. P. van, 1889. Animal parasites and messmates. Kegan, Paul \& Trench, London, $274 \mathrm{pp}$.

Bertin, L., 1958. Ecologie. In: Traité de zoologie. Publ. par. P. P. Grassé. Masson, Paris 13, $1885-1933$.

Bl.ACKBURn, M., 1948. Notes on some parasitic actinian larvae and on their host medusa in Australian waters. J. Coun. scient. ind. Res. Aust. 21, 183-189.

Bowman, T. E., Meyers, C. D. \& Hicks, S. D., 1963. Notes on associations between amphipods and medusae in Chesapeake and Narragansett Bays and the Niantic river. Chesapeake Sci. 4, 141-146.

Bresslav, E., 1928-1933. Turbellaria. In: Handbuch der Zoologie, Begr. von W. Kükenthal. De Gruyter, Berlin 2 (1), 54-320.

- 1932. Plathelminthes. In: Handwörterbuch der Naturwissenschaften. Fischer, Jena 7, 1105 bis 1138 .

- \& Retsinger, E., 1923. Plathelminthes, Vermes Amera, 1. Cladus. In: Handbuck der Zoologie. Begr. von W. KüKenTHaL. De Gruyter, Berlin 2 (1), 34-51.

Broch, H., 1928. Hydrozoa I. Tierwelt Nord-Ostsee 3b, 1-100.

BROWNE, E. T. 1895. Report on the medusae of the LBMC district. Proc. Trans. Liverpool biol. Soc. 9, 243-286.

BRUCE, A. J,, 1972. An association between a pontoniinid shrimp and a rhizostomatous scyphozoan. Crustaceana 23, 300-302.

Buchrolz, H. A., 1953. Die Wirtstiere des Amphipoden Hyperia galba in der Kieler Bucht. Faun. Mitt. Norddeutschland 3, 5-6.

Buchner, P., 1934. Symbiose. In: Handwörterbuch der Naturwissenschaften. Fischer, Jena 9, $775-789$.

Carlgren, O., 1932. Die Ceriantharien, Zoantharien und Actiniarien des arktischen Gebietes. Fauna arct. 6, 253-266.

- 1933. Actiniaria. Die Aktinienlarven und die Ceriantharienlarven. Nord. Plankt. (Zool.) 6, 65-78.

CAuliery, M., 1954. Parasitism and symbiosis. Sidwick \& Jackson, London, $340 \mathrm{pp}$.

Chun, C., 1896. Beiträge zur Kenntnis ostafrikanischer Medusen und Siphonophoren nach den Sammlungen Dr. Stuhlmanns. Mitt. naturhist. Mus. Hamb. 13, 1-19.

Corrington, J. L., 1927. Commensal association of a spider crab and a medusa. Biol. Bull. mar. biol. Lab., Woods Hole 53, 346-350. 
DAHL, E., 1959a. The amphipod Hyperia galba, an ectoparasite of the jellyfish Cyanea capillata. Nature, Lond. 183, 1749.

- 1959b. The hyperiid amphipod, Hyperia galba, a true ectoparasite on jellyfish. Univ. Bergen Arb. (Naturvit. rekke) 9, 1-8.

DAHL, F., 1893. Untersuchungen über die Tierwelt der Unterelbe. Ber. Kommn, wiss. Unters. dt. Meere 6, 529-593.

Dales, R. P., 1966. Symbiosis in marine organisms. In: Symbiosis. Ed. by S. H. Henry. Acad. Press, London 1, 299-326.

Della Valle, A., 1893. Gammarini del Golfo di Napoli. In: Fauna Flora Golf Neapel 20, 286-292.

Dendy, A., 1888. Note on some actinian larvae parasitic upon a medusa from Port Philipp. Trans. Proc. R. Soc. Victoria 15, 112.

Dollfuss, R. P., 1923. Enumération des cestodes du plancton et des invertébrés marins. Annls Parasit. hum. comp. 1, 276-300.

- 1929. Addendum à mon énumération des cestodes et des invertébrés. Annls Parasit. hum. comp. 7, 325-347.

- 1963. Liste des coelentérés marins palaearctiques et indien où ont été trouvés des trematodes digénetiques. Bull. Inst. Pêch. marit. Maroc 9-10, 33-57.

Eibl-Eibesfeldt, J., 1955. Uber Symbiose, Parasitismus und andere zwischenartiche Beziehungen tropischer Meeresfische. Z. Tierpsychol. 12, 203-219.

Ehrenbaum, E., 1927. Elasmobranchii. Tierwelt Nord-Ostsee 12e, 1-66.

Evans, F. \& Sheader, M., 1972. Host species of the hyperiid amphipod Hyperoche medusarum (Kroyer) in the North Sea. Crustaceana (Suppl.) 3, 275-276.

Evans, W. \& Ashworth, A., 1909. Some Medusae and Ctenophorae from the Firth of Forth. Proc. R. phys. Soc. Edinb. 17, 300-311.

FabER, F., 1829. Naturgeschichte der Fische Islands mit einem Anhange von den isländischen Medusen und Strahltieren. Frankfurt, 206 pp.

FEWKES, J. W., 1889. New invertebrates from the coast of California. Bull. Essex Inst. 21, 122.

Freycinet, L., 1833. Les zoophytes ou animaux rayonnés: des méduses. In: Voyage de l'Astrolabe pendant 1826-1829 (Zool.) 2, 559-573.

Fuhrmann, O., 1931. Cestoidea. In: Handbuch der Zoologie. Begr. von W. Kükenthal. De Gruyter, Berlin, 2 (1), 141-416.

Goormaghtigh, E. \& Parmentrer, M., 1973. Le crustacé amphipode Hyperia galba, parasite de la méduse Rbizostoma octopus. Naturalistes belg. 54, 131-135.

Graeffe, E., 1884. Ubersicht über die Seetierfauna des Golfes von Triest nebst Notizen über Vorkommen, Lebensweise, Erscheinen und Fortpflanzung der einzelnen Arten. Arb. zool. Inst. Univ. Wien 5, 333-362.

Gutsell, I. G., 1928: The spider crab, Libinia dubia, and the jellyfish Stomolopbus meleagris found associated at Beaufort (North Carolina). Ecology 9, 358-359.

HaAcke, W., 1886. Die Scyphomedusen des St. Vincent Golfes. Jena Z. Naturw. 8, 589-638.

HaAthela, I. \& LAssig, J., 1967. Record of Cyanea capillata (Scyphozoa) and Hyperia galba (Amphipoda) from the Gulf of Finland and the Northern Baltic. Annls zool. fenn. 4, 469 to 471 .

Hale, H. M., 1952. Isopoda, Families Cymothoidae and Serolidae. Rep. B. A. N. Z. Antarctic Res. Exped., 1929-1931 6 (2), 21-36.

Hayashi, K. \& Miyake, S., 1968. Three caridean shrimps associated with a medusa from Tanabe Bay, Japan. Publ. Seto mar. biol. Lab. 16, 11-19.

Hollowday, E. D., 1947. On the commensal relationship between amphipod Hyperia galba (MonT.) and the Scyphomedusa Rbizostoma pulmo var. octopus. J. Quekett microsc. Club 4, 187-190.

Holthuis, L. B., 1947. The Decapoda of the Siboga Expedition pt. IX: The Hippolytidae and Rhynchocinetidae collected by the Siboga and Snellius Expeditions with remarks on other species. Siboga Exped. 39a, 1-100. 
Humes, A. G., 1963. Two new semiparasitic harpacticoid copepods from the coast of New Hamshire. J. Wash. Acad. Sci. 43, 360-373.

Jachowsкi, R., 1974. Observations on the moon-jelly, Aurelia aurita, and the spider crab, Libinia dubia. Chesapeake Sci. 4, 195.

KäNDLER, R., 1950. Jahreszeitliches Vorkommen und unperiodisches Auftreten von Fischbrut, Medusen und Decapodenlarven im Fehmarnbelt in den Jahren 1934-1943. Ber. dt. wiss. Kommn. Meeresforsch. 12, 49-85.

- 1961. Uber das Vorkommen von Fischbrut, Decapodenlarven und Medusen in der Kieler Förde. Kieler Meeresforsch. 17, 48-64.

Krshinouye, K., 1902. Some new scyphomedusae of Japan. J. Coll. Sci. imp. Univ. Tokyo 17 (7), 1-17.

- 1910. Some medusae of Japanese waters. J. Coll. Sci. imp. Univ. Tokyo 27 (9), 1-35.

Kotzebue, O., 1830. Neue Reise um die Welt in den Jahren 1823,1824, 1825, 1826 auf der Brigg "Rurik". Weimar, 1-2.

Kramp, P. L., 1956. Pelagic Fauna. pp. 65-86. In: The Galathea Deep Sea Expedition, 1950 to 1952. Ed. by A. F. Brunn, S. V. Greve, H. Mielche \& R. Spärck. Allen \& Unwin, London, $296 \mathrm{pp}$.

- 1968a. The Scyphomedusae collected by the Galathea Expedition 1950-1952. Vidensk. Meddr. dansk nath. Foren 131, 67-98.

- 1968b. Medusae in the Peru current system. Vidensk. Meddr. dansk. nath. Foren. 131, 199-208.

Krumbach, T., 1925. Scyphozoa. In: Handbuch der Zoologie. Begr. von W. Kükenthal. De Gruyter, Berlin, 1, 664-686.

Kuво, I., 1965. Macrura. In: New illustrated encyclopedia of the fauna of Japan. Hrsg. von Y. K. OKada, T. Uchida (u. a.). Hokuryukan, Tokyo 3, 591-629 (Japan.).

KüNNE, C., 1948. Medusen als Transportmittel für Aktinienlarven. Natur Volk 78, 174-176.

Kyle, H. M. \& Ehrenbaum, E., 1927. Teleostei physostomi. Tierwelt Nord-Ostsee 12f, 1-86.

Lane, F., 1957. Kingdom of the Octopus, the life history of the Cephalopoda. Jarrolds, London, $287 \mathrm{pp}$.

Laval, P., 1972. Comportement, parasitisme et écologie d'Hyperia schizogeneios STEBB. (Amphipode Hyperidae) dans le plancton de Villefranche sur mer. Annls Inst. océanogr., Monaco. 48, 49-74.

Light, S. F., 1914. Some Philippine Scyphomedusae, including two new genera, five new species and one new variety. Philipp. J. Sci. (D) 9, 195-231.

Linton, E, 1925. Notes on cestodes parasites of sharks and skates. Proc. U. S. natn. Mus. 64 (21), 1-114.

LrNstow, O. F. von, 1878. Compendium der Helminthologie. Hannover.

- 1903. Helminthologische Beobachtungen. Zbl. Bakt. Parasitkde 34, 520-531.

Liv, I. Y., 1955. Economic shrimps and prawns of northern China. Bull. mar. biol. Inst. Acad. Sci. Peking 4, 1-73.

McIntosh, W. C. \& Prince, E. E., 1890. On the development and life histories of the teleostean food and other fishes. Trans. R. Soc. Edinb. 35, 665-946.

MAN, J. G. DE, 1929. On a small collection of Decapoda, one of which, a Crangon, caught by the Danish Pacific Expedition at Jolo Islands, is new to science. Papers from Dr. Th. Mortensen's Pacific Expedition. 1914-1916. Vidensk. Meddr dansk. naturhist. Foren. 87, 105-134.

Meinert, F., 1890. Crustacea Malacostraca. In: Petersen's Det Vidensk. Udbytte "Haughs Togter" i de Dansk Have indenfor Skagen 1883-1886, 149-232.

Merxner, J., 1926. System der Turbellaria-Rhabdocoela II. Z. Morph. Ókol. Tiere 5, 577-624.

- 1928. Turbellaria. In: Tierwelt Nord-Ostsee 4b, 1-146.

Menon, M. G. K., 1930. The Scyphomedusae of Madras and neighbouring coast. Bull. Madras Govt. Mus. (Nat. Hist.) 3, 1-28.

Menzies, R. I. \& Dow, T., 1958. The largest known bathypelagic isopod Anuropus bathypelagicus. Ann. Mag. nat. Hist. (Ser. 13) 1, 1-6. 
Metz, P., 1967. On the relation between Hyperia galba Montagu (Amphipoda Hyperiidae) and its host Aurelia aurita in the Isefjord-area (Sjaelland, Denmark). Vidensk. Meddr dansk. naturh. Foren. 130, 85-108.

Meyer, H. A. \& Möвıus, K., 1862. Kurzer Uberblick der in der Kieler Bucht von uns beobachteten wirbellosen Tiere als Vorläufer einer Fauna derselben. Arch. Naturgesch. 28, 229-237.

Moestafa, S. H. \& Mc Connaughey, B. H., 1966. Catostylus ouwensi (Rhizostomae Catostylidae), a new jellyfish from Irian (New Guinea) and Ouwensia catostyli ng. nsp. parasitic in C. ouwensi. Treubia 27, 1-9.

Monticelli, F. S., 1896. Appunti siu Cestadaria. Atti Accad. Sci. fis. mat., Napoli (Ser. 2) 5, 1892.

- 1897. Adelotacta Zoologica I, Pemmatodiscus sozialis n. sp. Mitt. Zool. Stn. Neapel 12, 432-462.

Moreira, M. G. B., 1961. Sobre Mastigias scintillae sp. nov. (Scyph. Rhiz.) das costas do Brasil. Bolm. Inst. Oceanogr., S Paulo 11 (2), 5-29.

Müller, F., 1860. On philomedusa, a parasite on medusa. Ann. Mag. nat. Hist. (Ser. 3) 6, 432.

Orton, J. H., 1922. The mode of feeding of the jellyfish Aurelia aurita on smaller organisms in the plankton. Nature, Lond. 110, 178-179.

Panikar, N. K. \& Prasad, R. R., 1952. On an interesting association of ophiurids, fish and crabs with the jellyfish Rhopilema hispidum. J. Bombay nat. Hist. Soc. 51, 295-296.

Pérès, J. M., 1958. Trois plongées dans le canyon du Cap sicié effectuées avec le bathyscaph F. N. R. S. III de la Marine Nationale. Bull. Inst. océanogr. Monaco 1115, 1-21.

Perkins, H. F., 1908. Note on the occurrence of Cassiopea xamachana and Polyclonia frondosa at the Tortugas. Publs. Carneg. Instn. 102, 150-152.

Phillips, P. J., 1973. The occurrence of the remarkable scyphozoan Deepstaria enigmatica, in the Gulf of Mexiko and some observations on cnidarian symbionts. Gulf Res. Rep. 4, 166168.

- \& Levin, L., 1973. Cestode larvae from Scyphomedusae of the Gulf of Mexico. Bull. mar. Sci. 23, 574-584.

-, BURKE, W. D. \& KeENER, E. J., 1969. Observations on the trophic significance of jellyfishes in Mississippi Sound with quantitative data on the associative behaviour of small fishes with medusae. Trans. Am. Fish. Soc. 98, 703-712.

PiRLot, I. M., 1932. Introduction à l'étude des amphipodes hyperides. Annls Inst. océanogr., Monaco 12, 1-36.

Prell, H., 1909. Beiträge zur Kenntnis der Lebensweise einiger Pantopoden. Bergens Mus. Arb. 1909 (10), 1-30.

Rao, H. S., 1931. Notes on Scyphomedusae in the Indian Museum. Rec. Ind. Mus. 33, 25-62.

Rathbun, M. J., 1925. The spider crabs of America. Bull. U. S. natn. Mus. 129, 1-598.

Rathke, H., 1836. Beitrag zur Fauna der Krym. Mém. Acad. Imp. Sci. St. Pétersburg, Mém. sav. Etrang. 3, 424.

ReDdiah, K., 1968. Three new species of Paramacrochiron (Lichomolgidae) associated with medusae. Crustaceana (Suppl.) 1, 193.

Romanes, G. J., 1876. An account of some new species, varieties and monstrous forms of medusae. J. Linn. Soc. (Zool.) 12, 524-531.

Russel, F. S., 1967. On a remarkable new scyphomedusan Deepstaria enigmatica. J. mar. biol. Ass. U. K. 47, 469-473.

SARS, G. O., 1879a. Indberetning til Departementet for det Indre om de af ham in Aarene 1864-1878 anstillede undersøgelser angaanende Saltvands fiskierne. Christiania, 221 pp.

- 1879b. Report of practical and scientific investigations of cod fisheries near the Loffoten Islands 1864-1869 and 1870-1873. Rept. U. S. Commnr fish. 1877, 612-661.

Schellenberg, A., 1927. Amphipoda des nordischen Planktons. Nord. Plankt. (Zool.) 3, 589 bis 722.

Schnakenbeck, W., 1925. Heterosomata. Tierwelt Nord-Ostsee $\mathbf{1 2} \mathbf{h}_{1}, \mathbf{1}-60$.

— 1926. Teleostei Physoclisti 6. Gadiformes. Tierwelt Nord-Ostsee 12ge, 45-88.

- 1927. Cyclostomi. Tierwelt Nord-Ostsee 12 d, 1-14.

Schultze, F. S., 1898. Rhizostomeen von Ternate. Abh. Senckenberg. naturf. Ges. 24, 153-165. 
ShoJima, Y., 1963. Scyllarid phyllosomas' habit of accompanying the jellyfish. Bull. Jap. Soc. scient. Fish. 29, 349-353.

Sims, H. W. jr. \& BROwN, C. L. jr., 1968. A giant scyllarid phyllosoma-larva taken north of Bermuda (Palinuridea). Crustaceana (Suppl.) 2, 80-82.

Southwell, T., 1921. On a larval cestode from the umbrella of a jellyfish. Mem. Indian Mus. $5,559-562$.

Sprehn, C., 1953. Trematoda. Tierwelt Nord-Ostsee 4c, 1-60.

- 1934. Cestoidea. Tierwelt Nord-Ostsee 4c2, 61-91.

- 1938. Lehrbuch der Helminthologie. Bornträger, Berlin, 998 pp.

Stephensen, K., 1927. Amphipoda. Tierwelt Nord-Ostsee 10 f, 1-188.

Steuer, A., 1910. Planktonkunde. Teubner, Leipzig, 723 pp.

Stiasny, G., 1919. Die Scyphomedusensammlung des Naturhistorischen Reichsmuseums in Leiden, III. Rhizostomae. Zoöl. Meded., Leiden 5, 213-230.

- 1921. Studien über Rhizostomeen mit besonderer Berücksichtigung der Fauna des Malayischen Archipels nebst einer Revision des Systems. Capita Zool. 1 (2), 1-179.

StUnKard, H. W., 1967. The life cycle and developmental stages of a digenetic trematode, whose unencysted metacercarial stages occur in medusae. Biol. Bull. mar. biol. Lab., Woods Hole 133, 488.

- 1968. Studies on the life-history of Distomum pyriforme Linton 1900. Biol. Bull. mar. biol. Lab., Woods Hole 135, 439.

- 1969. The morphology and life-history of Neopechona pyriforme (LinTon) ng. n. comb. (Trematoda, Lepocreadidae). Biol. Bull. mar. biol. Lab., Woods Hole 136, 96-113.

Suzuki, K., 1965. On a young crab found near the oral arms of the jellyfish Rhopilema esculenta Kish. Researches on Crustacea 2, 77-82 (Japan. m. engl. Zsfg.).

TAtTersall, W. M., 1906. The marine fauna of the coast of Ireland VIII. Pelagic Amphipoda of the Irish Atlantic Slope. Rep. Sea inld. Fish. Ire, 63, 99.

- 1915. Die nordischen Isopoden. Nord. Plankt. (Zool.) 3, 181-310.

Thiel, H., 1970. Scyphozoa. In: The encyclopedia of the biological science. Van Nostrand Reinhold, New York, 830-836.

THies, M. E., 1964. Untersuchungen über die Ernährung und den Nahrungskreislauf bei Rbizostoma octopus L. Ag. Mitt. zool. StInst. Hamb. 61 (Erg. Bd), 247-269.

- 1966. Untersuchungen über die Herkunft, das Auftreten, das Wachstum und die Fortpflanzung von Rhizostoma octopus. L. Ag. im Elbmündungsgebiet. Abh. Verh. naturwiss. Ver. Hamburg (NF) 10, 59-88.

- 1970a. Das Zusammenleben von Jung- und Kleinfischen mit Rhizostomeen. Ber. dt. wiss. Kommn. Meeresforsch. 21, 444-473.

- 1970b. Über das stoßweise Schwimmen eines Schwarmes von Caranx leptolepis (Cuv. ET Val.) (Pisces) synchron mit dem Schlagrhythmus der Meduse Acromitus flagellatus Stiasny. Abh. Verh. naturw. Ver. Hamburg 14, 71-79.

- 1971. Tintenfische "reiten" auf Quallen. Abh. Verh. naturwiss. Ver. Hamburg 15, 37-40.

Thомas, L. R., 1963. Phyllosoma-larvae associated with medusae. Nature, Lond. 198, 208.

TRотт, L. B., 1972. The portunid crab Charybdis feriatus (L.) commensal with the scyphomedusan jellyfish Stomolopbus nomurai (KISH.) in Hongkong. Crustaceana 23, 305-306.

UCHIDA, T., 1926. The anatomy and development of a rhizostome medusa, Mastigias papua L. AG., with observations on the phylogeny of Rhizostomae. J. Fac. Sci. imp. Univ. Tokio (Sect. 4: Zool.) 1, 45-95.

- \& Hanaoka, K. I., 1933. On the morphology of a stalked medusa, Thaumatoscyphus distinctus KIsH. J. Fac. Sci. Hokkaido imp. Univ. (Ser. 6: Zool.) 2, 135-153.

VADER, W., 1972. Associations between gammarid and caprellid amphipods and medusae. Sarsia 50, 51-56.

Vallentin, R., 1888. Psorospermium lucernariae. Zool. Anz. 11, 622-623.

Vannucci, M., 1951. Hydrozoa e Scyphozoa existentes no Instituto Paulista de Oceanografia I. Bolm. Inst. paul. Oceanogr. 2, 69-100.

- 1954. Hydrozoa e Scyphozoa existentes no Instituto Oceanografico II. Bolm. Inst. Oceanogr. S Paulo 5, 95-149. 
- \& Mendes, M., 1944. Sobre a larva de Dibothriorbynchus dinoi n. sp. parasita dos Rhizostomata (Cest. Tetrarhynchidea). Archs Mus. parana. 4, 47-82.

VERWEY, J., 1942. Die Periodizität im Auftreten und die aktiven und passiven Bewegungen der Quallen. Archs. néerl. Zool. 6, 365-468.

Vescovi, P. DE, 1895. Biologici observationes circa aliquot hospites qui in Medusa Rbizostoma pulmo LinN. inveniuntur. Zool. Res. 1, 37-40.

Westernhagen, H. von, 1976. Some aspects of the biology of the hyperiid amphipod Hyperoche medusarum. Helgoländer wiss. Meeresunters. 28, 43-50.

Weymouth, F. W., 1910. Synopsis of the true crabs (Brachyura) of Monterey Bay, California. Leland Stanford jr. Univ. Publs. Univ. Ser. 4, 42-106.

WhILE, M. G. \& BORSE, D. G., 1972. The interrelationship of Hyperia galba (Crustacea Amphipoda) and Desmonema gaudichaudi (Scyphomedusae Semaeostomae) from the Antarctic. Br. Antarct. Surv. Bull. 27, 39-49.

Williamson, H. C., 1915. Crustacea Decapoda, Larven. Nord. Plankt. (Zool.) 3, 315-588.

WiLsmore, L. J., 1911. On some Hexactiniae from New South Wales. J. Linn. Soc. (Zool.) 32, $39-57$.

YANEZ, A. P., 1915. Observacion de larvas medusofilas de Actinie, Rev. Biol. mar. 3, 231-232.

Anschritt des Verfassers: Dr. M. E. THIEL

Brunsdorfer Weg 2

D-2000 Hamburg 67

Bundesrepublik Deutschland 